\title{
Syzygies of Prym and paracanonical curves of genus 8
}

\author{
Elisabetta Colombo, Gavril Farkas, Alessandro Verra, and Claire Voisin
}

\begin{abstract}
By analogy with Green's Conjecture on syzygies of canonical curves, the PrymGreen conjecture predicts that the resolution of a general level $p$ paracanonical curve of genus $g$ is natural. The Prym-Green Conjecture is known to hold in odd genus for almost all levels. Probabilistic arguments strongly suggested that the conjecture might fail for level 2 and genus 8 or 16. In this paper, we present three geometric proofs of the surprising failure of the Prym-Green Conjecture in genus 8, hoping that the methods introduced here will shed light on all the exceptions to the Prym-Green Conjecture for genera with high divisibility by 2 .
\end{abstract}

Keywords. Paracanonical curve; syzygy; genus 8; moduli of Prym varieties

2010 Mathematics Subject Classification. 13D02; 14H10

\section{[Français]}

Titre. Syzygies de Prym et courbes paracanoniques de genre 8

Résumé. Par analogie avec la conjecture de Green sur les syzygies des courbes canoniques, la conjecture de Prym-Green prédit que la résolution d'une courbe générale, paracanonique, de genre $g$ et de niveau $p$ est naturelle. Cette conjecture est connue en genre impair pour presque tout niveau. Des arguments probabilistes ont fortement suggéré qu'elle pourrait s'avérer fausse pour le niveau 2 en genre 8 et 16 . Dans cet article, nous présentons trois démonstrations géométriques de la surprenante non-validité de la conjecture de Prym-Green en genre 8, en espérant que les méthodes introduites apporteront un éclairage nouveau sur toutes les exceptions à la conjecture de Prym-Green pour des genres divisibles par une grande puissance de 2 .

Received by the Editors on December 15, 2016, and in final form on June 23, 2017.

Accepted on June 27, 2017.

Elisabetta Colombo

Università di Milano, Dipartimento di Matematica, Via Cesare Saldini 50, 20133 Milano, Italy

e-mail: elisabetta.colombo@unimi.it

\section{Gavril Farkas}

Humboldt-Universität zu Berlin, Institut für Mathematik, Unter den Linden 6, 10099 Berlin, Germany e-mail: farkas@math.hu-berlin.de

\section{Alessandro Verra}

Università Roma Tre, Dipartimento di Matematica, Largo San Leonardo Murialdo, 1-00146 Roma, Italy

e-mail:verra@mat.uniroma3.it

Claire Voisin

Collége de France 3, Rue d'Ulm, 75005 Paris, France

e-mail: claire.voisin@imj-prg.fr 


\section{Contents}

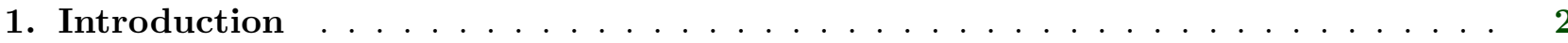

2. Syzygies of paracanonical curves of genus $8 \ldots \ldots \ldots \ldots$

3. First proof: reducible spin curves $\ldots \ldots \ldots \ldots \ldots$

4. Second proof: Divisor class calculations on $\overline{\mathcal{R}}_{g} \ldots \ldots \ldots \ldots$

5. Rank 2 vector bundles and singular quartics $\ldots \ldots \ldots \ldots$

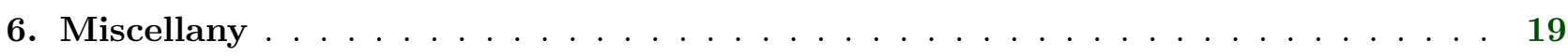

\section{Introduction}

By analogy with Green's Conjecture on the syzygies of a general canonical curve [18], [19], the PrymGreen Conjecture, formulated in [10] and [3], predicts that the resolution of a paracanonical curve

$$
\phi_{K_{C} \otimes \eta}: C \hookrightarrow \mathbf{P}^{g-2},
$$

where $C$ is a general curve of genus $g$ and $\eta \in \operatorname{Pic}^{0}(C)[\ell]$ is an $\ell$-torsion point is natural. For even genus $g=2 i+6$, the Prym-Green Conjecture amounts to the vanishing statement

$$
K_{i, 2}\left(C, K_{C} \otimes \eta\right)=K_{i+1,1}\left(C, K_{C} \otimes \eta\right)=0,
$$

in terms of Koszul cohomology groups. Equivalently, the genus $g$ paracanonical level $\ell$ curve $C \subseteq \mathbf{P}^{g-2}$ satisfies the Green-Lazarsfeld property $\left(N_{i}\right)$. The Prym-Green Conjecture has been proved for all odd genera $g$ when $\ell=2$, see [8], or $\ell \geq \sqrt{\frac{g+2}{2}}$, see [9]. For even genus, the Prym-Green Conjecture has been established by degeneration and using computer algebra tools in [3] and [4], for all $\ell \leq 5$ and $g \leq 18$, with two possible mysterious exceptions in level 2 and genus $g=8,16$ respectively. The last section of [3] provides various pieces of evidence, including a probabilistic argument, strongly suggesting that for $g=8$, one has $\operatorname{dim} K_{1,2}\left(C, K_{C} \otimes \eta\right)=1$, and thus the vanishing (1.1) fails in this case. It is tempting to believe that the exceptions $g=8,16$ can be extrapolated to higher genus, and that for genera $g$ with high divisibility by 2 , there are genuinely novel ways of constructing syzygies of Prym-canonical curves waiting to be discovered. It would be very interesting to test experimentally the next relevant case $g=24$. Unfortunately, due to memory and running time constraints, this is currently completely out of reach, see [3] and [7].

The aim of this paper is to confirm the expectation formulated in [3] and offer several geometric explanations for the surprising failure of the Prym-Green Conjecture in genus 8, hoping that the geometric methods described here for constructing syzygies of Prym-canonical curves will eventually shed light on all the exceptions to the Prym-Green Conjecture. We choose a general Prym-canonical curve of genus 8

$$
\phi_{K_{C} \otimes \eta}: C \hookrightarrow \mathbf{P}^{6},
$$

with $\eta^{\otimes 2}=\mathcal{O}_{C}$. Set $L:=K_{C} \otimes \eta$ and denote $I_{C, L}(k):=\operatorname{Ker}\left\{\operatorname{Sym}^{k} H^{0}(C, L) \rightarrow H^{0}\left(C, L^{\otimes k}\right)\right\}$ for all $k \geq 2$. Observe that $\operatorname{dim} I_{C, L}(2)=\operatorname{dim} K_{1,1}(C, L)=7$ and $\operatorname{dim} I_{C, L}(3)=49$, therefore as $[C, \eta]$ varies in moduli, the multiplication map

$$
\mu_{C, L}: I_{C, L}(2) \otimes H^{0}(C, L) \rightarrow I_{C, L}(3)
$$

globalizes to a morphism of vector bundles of the same rank over the stack $\mathcal{R}_{8}$ classifying pairs $[C, \eta]$, where $C$ is a smooth curve of genus 8 and $\eta \in \operatorname{Pic}^{0}[2] \backslash\left\{\mathcal{O}_{C}\right\}$. 
Theorem 1. For a general Prym curve $[C, \eta] \in \mathcal{R}_{8}$, one has $K_{1,2}(C, L) \neq 0$. Equivalently the multiplication map $\mu_{C, L}: I_{C, L}(2) \otimes H^{0}(C, L) \rightarrow I_{C, L}(3)$ is not an isomorphism.

We present three different proofs of Theorem 1. The first proof, presented in Section 3 uses the structure theorem already pointed out in [3] for degenerate syzygies of paracanonical curves in $\mathbf{P}^{6}$. Precisely, if a paracanonical genus 8 curve $\phi_{K_{C} \otimes \eta}: C \hookrightarrow \mathbf{P}^{6}$, where $\eta \neq \mathcal{O}_{C}$, has a syzygy $0 \neq \gamma \in K_{1,2}\left(C, K_{C} \otimes \eta\right)$ of sub-maximal rank (see Section 2 for a precise definition), then the syzygy scheme of $\gamma$ consists of an isolated point $p \in \mathbf{P}^{6} \backslash C$ and a residual septic elliptic curve $E \subseteq \mathbf{P}^{6}$ meeting $C$ transversally along a divisor $e$ of degree 14 , such that if $e$ is viewed as a divisor on $C$ and $E$ respectively, then

$$
e_{C} \in\left|K_{C} \otimes \eta^{\otimes 2}\right| \quad \text { and } \quad e_{E} \in\left|\mathcal{O}_{E}(2)\right| .
$$

The union $D:=C \cup E \hookrightarrow \mathbf{P}^{6}$, endowed with the line bundle $\mathcal{O}_{D}(1)$ is a degenerate spin curve of genus 22 in the sense of [5]. The locus of stable spin structures with at least 7 sections defines a subvariety of codimension $21=\left(\begin{array}{l}7 \\ 2\end{array}\right)$ inside the moduli space $\overline{\mathcal{S}}_{22}^{-}$of stable odd spin curves of genus 22. By restricting this condition to the locus of spin structures having $D:=C \cup_{e} E$ as underlying curve, it turns out that one has enough parameters to realize this condition for a general $C \subseteq \mathbf{P}^{6}$ if and only if

$$
\operatorname{dim}\left|K_{C} \otimes \eta^{\otimes 2}\right|=7,
$$

which happens precisely when $\eta^{\otimes 2} \cong \mathcal{O}_{C}$. Therefore for each Prym-canonical curve $C \subseteq \mathbf{P}^{6}$ of genus 8 there exists a corresponding elliptic curve $E \subseteq \mathbf{P}^{6}$ such that the intersection divisor $E \cdot C$ verifies (1.2), which forces $K_{1,2}\left(C, K_{C} \otimes \eta\right) \neq 0$.

The second and the third proofs involve the reformulation given in Section 2.B (see Proposition 5 ) of the condition that a paracanonical curve $\phi_{L}: C \hookrightarrow \mathbf{P}^{6}$ have a non-trivial syzygy. Precisely, if $\phi_{L}(C)$ is scheme-theoretically generated by quadrics, then $K_{1,2}(C, L) \neq 0$, if and only if there exists a quartic hypersurface in $\mathbf{P}^{6}$ singular along $C \subseteq \mathbf{P}^{6}$, which is not a quadratic polynomial in quadrics vanishing along $C$, that is, it does not belong to the image of the multiplication map

$$
\operatorname{Sym}^{2} I_{C, L}(2) \rightarrow I_{C, L}(4) \text {. }
$$

Equivalently, one has $H^{1}\left(\mathbf{P}^{6}, \mathcal{I}_{C / \mathbf{P}^{6}}^{2}(4)\right) \neq 0$.

The second proof presented in Section 4 uses intersection theory on the stack $\overline{\mathcal{R}}_{8}$. The virtual Koszul divisor of Prym curves $[C, \eta] \in \mathcal{R}_{8}$ having $K_{1,2}\left(C, K_{C} \otimes \eta\right) \neq 0$, splits into two divisors $\mathfrak{D}_{1}$ and $\mathfrak{D}_{2}$ respectively, corresponding to the case whether $C \subseteq \mathbf{P}^{6}$ is not scheme-theoretically cut out by quadrics, or $H^{1}\left(\mathbf{P}^{6}, \mathcal{I}_{C / \mathbf{P}^{6}}^{2}(4)\right) \neq 0$ respectively. We determine the virtual classes of both closures $\overline{\mathfrak{D}}_{1}$ and $\overline{\mathfrak{D}}_{2}$. Using an explicit uniruled parametrization of $\overline{\mathcal{R}}_{8}$ constructed in [11], we conclude that the class $\left[\overline{\mathfrak{D}}_{2}\right] \in C H^{1}\left(\overline{\mathcal{R}}_{8}\right)$ cannot possibly be effective (see Theorem 20 ). Therefore, again $K_{2,1}\left(C, K_{C} \otimes \eta\right) \neq 0$, for every Prym curve $[C, \eta] \in \mathcal{R}_{8}$.

The third proof given in Section 5 even though subject to a plausible, but still unproved transversality assumption, is constructive and potentially the most useful, for we feel it might offer hints to the case $g=16$ and further. The idea is to consider rank 2 vector bundles $E$ on $C$ with canonical determinant and $h^{0}(C, E)=h^{0}(C, E(\eta))=4$. (Note that the condition that $\eta$ is 2-torsion is equivalent to the fact that $E(\eta)$ also has canonical determinant, which is essential for the existence of such nonsplit vector bundles, cf. [15].) By pulling back to $C$ the determinantal quartic hypersurface consisting of rank 3 tensors in

$$
\mathbf{P}\left(H^{0}(C, E)^{\vee} \otimes H^{0}(C, E(\eta))^{\vee}\right) \cong \mathbf{P}^{15}
$$

under the natural map $H^{0}\left(C, K_{C} \otimes \eta\right)^{\vee} \rightarrow H^{0}(C, E)^{\vee} \otimes H^{0}(C, E(\eta))^{\vee}$, we obtain explicit quartic hypersurfaces singular along the curve $C \subseteq \mathbf{P}^{6}$. Our proof that these are not quadratic polynomials 
into quadrics vanishing along the curve, that is, they do not lie in the image of $\operatorname{Sym}^{2} I_{C, L}(2)$ remains incomplete, but there is a lot of evidence for this.

The methods of Section 5 suggests the following analogy in the next case $g=16$. If $[C, \eta] \in \mathcal{R}_{16}$ is a Prym curve of genus 16, there exist vector bundles $E$ on $C$ with $\operatorname{det} E \cong K_{C}$ and satisfying $h^{0}(C, E)=h^{0}(C, E(\eta))=6$. Potentially they could be used to prove that $K_{5,2}\left(C, K_{C} \otimes \eta\right) \neq 0$ and thus confirm the next exception to the Prym-Green Conjecture.

\section{Syzygies of paracanonical curves of genus 8}

Let $C$ be a general smooth projective curve of genus 8. For a non-trivial line bundle $\eta \in \operatorname{Pic}^{0}(C)$, we shall study the paracanonical line bundle $L:=K_{C} \otimes \eta$. When $\eta$ is a 2-torsion point, we speak of the Prym-canonical line bundle $L$. For each paracanonical bundle $L$, we have $h^{0}(C, L)=7$ and an induced embedding

$$
\phi_{L}: C \hookrightarrow \mathbf{P}^{6} .
$$

The goal is to understand the reasons for the non-vanishing of the Koszul group $K_{1,2}(C, L)$ of a Prym-canonical bundle $L$, as suggested experimentally by the results of [3], [4].

Let $I_{C}(2)=I_{C, L}(2) \subseteq H^{0}\left(\mathbf{P}^{6}, \mathcal{O}_{\mathbf{P}^{6}}(2)\right)$, respectively $I_{C}(3)=I_{C, L}(3) \subseteq H^{0}\left(\mathbf{P}^{6}, \mathcal{O}_{\mathbf{P}^{6}}(3)\right)$ be the ideal of quadrics, respectively cubics, vanishing on $\phi_{L}(C)$. It is well-known that whenever $L$ is projectively normal, the non-vanishing of the Koszul cohomology group $K_{1,2}(C, L)$ is equivalent to the non-surjectivity of the multiplication map

$$
\mu_{C, L}: H^{0}\left(\mathbf{P}^{6}, \mathcal{O}_{\mathbf{P}^{6}}(1)\right) \otimes I_{C}(2) \rightarrow I_{C}(3) .
$$

Note that

$$
\operatorname{dim} I_{C}(2)=\left(\begin{array}{l}
8 \\
2
\end{array}\right)-21=7, \quad \text { and } \quad \operatorname{dim} I_{C}(3)=\left(\begin{array}{l}
9 \\
3
\end{array}\right)-3 \cdot 14+7=49,
$$

respectively, so that the two spaces appearing in the map (2.3) have the same dimension. Denote by $P_{8}^{14}$ the universal degree 14 Picard variety over $\mathcal{M}_{8}$ consisting of pairs $[C, L]$, where $[C] \in \mathcal{M}_{8}$ and $L \neq K_{C}$. The jumping locus

$$
\mathfrak{K} \mathfrak{o} \mathfrak{s} \mathfrak{z}:=\left\{[C, L] \in P_{8}^{14}: K_{1,2}(C, L) \neq 0\right\}
$$

is a divisor. It turns out, cf. Theorem 5.3 of [3] and Proposition 8, that $\mathfrak{K} 0 \mathfrak{s} \mathfrak{z}$ splits into two components depending on the rank of the corresponding non-zero syzygy from $K_{1,2}(C, L)$.

Definition 2. The rank of a non-zero syzygy $\gamma=\sum_{i=0}^{6} \ell_{i} \otimes q_{i} \in \operatorname{Ker}\left(\mu_{C, L}\right)$ is the dimension of the subspace $\left\langle\ell_{0}, \ldots, \ell_{6}\right\rangle \subseteq H^{0}\left(\mathbf{P}^{6}, \mathcal{O}_{\mathbf{P}^{6}}(1)\right)$. The syzygy scheme $\operatorname{Syz}(\gamma)$ of $\gamma$ is the largest subscheme $Y \subseteq \mathbf{P}^{6}$ such that $\gamma \in H^{0}\left(\mathbf{P}^{6}, \mathcal{O}_{\mathbf{P}^{6}}(1)\right) \otimes I_{Y}(2)$.

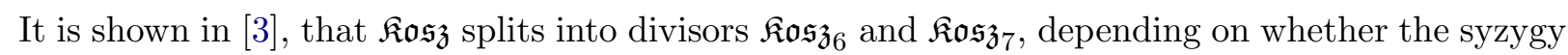
$0 \neq \gamma \in \operatorname{Ker}\left(\mu_{C, L}\right)$ has rank 6 or 7 respectively. By a specialization argument to irreducible nodal curves, it follows from [3] that $\mathcal{R}_{8} \nsubseteq \mathfrak{K o s}_{\mathfrak{z}_{7}}$. A direct, more transparent proof of this fact will be given in Proposition 13.

\section{A. Paracanonical curves of genus 8 with special syzygies and elliptic curves}

We summarize a few facts already stated or recalled in Section 5 of [3] concerning rank 6 syzygies of paracanonical curves in $\mathbf{P}^{6}$. Very generally, let

$$
\gamma=\sum_{i=1}^{6} \ell_{i} \otimes q_{i} \in H^{0}\left(\mathbf{P}^{6}, \mathcal{O}_{\mathbf{P}^{6}}(1)\right) \otimes H^{0}\left(\mathbf{P}^{6}, \mathcal{O}_{\mathbf{P}^{6}}(2)\right)
$$


be a rank 6 linear syzygy among quadrics in $\mathbf{P}^{6}$. The linear forms $\ell_{1}, \ldots, \ell_{6}$ define a point $p \in \mathbf{P}^{6}$. Following Lemma 6.3 of [16], there exists a skew-symmetric matrix of linear forms $A:=\left(a_{i j}\right)_{i, j=1, \ldots, 6}$, such that

$$
q_{i}=\sum_{j=1}^{6} \ell_{j} a_{i j} .
$$

In the space $\mathbf{P}^{20}$ with coordinates $\ell_{1}, \ldots, \ell_{6}$ and $a_{i j}$ for $1 \leq i<j \leq 6$, one considers the 15 -dimensional variety $X_{6}$ defined by the 6 quadratic equations $\sum_{j=1}^{6} \ell_{j} a_{i j}=0$, where $i=1, \ldots, 6$ and by the cubic equation $\operatorname{Pfaff}(A)=0$ in the variables $a_{i j}$. The original space $\mathbf{P}^{6}$ embeds in $\mathbf{P}^{20}$ via evaluation. The syzygy scheme $\operatorname{Syz}(\gamma)$ is the union of the point $p$ and of the intersection $D$ of $\mathbf{P}^{6}$ with the variety $X_{6}$. It follows from Theorem 4.4 of [6], that for a general rank 6 syzygy $\gamma$ as above, $D \subseteq \mathbf{P}^{6}$ is a smooth curve of genus 22 and degree 21 such that $\mathcal{O}_{D}(1)$ is a theta characteristic.

In the case at hand, that is, when $[C, L] \in \mathfrak{K}_{0} \mathfrak{s}_{6}$, the curve $D$ must be reducible, for it has $C$ as a component. More precisely:

Lemma 3. For a general paracanonical curve $C \subseteq \boldsymbol{P}^{6}$ having a rank 6 syzygy, the curve $D$ is nodal and consists of two components $C \cup E$, where $E \subseteq \boldsymbol{P}^{6}$ is an elliptic septic curve. Furthermore, $\mathcal{O}_{D}(2)=\omega_{D}$. The intersection $e:=C \cdot E$, viewed as a divisor on $C$ satisfies $e_{C} \in\left|\mathcal{O}_{C}(2) \otimes K_{C}^{\vee}\right|$, and as a divisor on $E$, satisfies $e_{E} \in\left|\mathcal{O}_{E}(2)\right|$.

Remark 4. Note that $C$ is Prym-canonical or canonical if and only if $e_{C} \in\left|K_{C}\right|$.

The construction above is reversible. Firstly, general element $[C, L] \in \mathfrak{K}_{\mathfrak{o s}} \mathfrak{z}_{6}$ can be reconstructed as the residual curve of a reducible spin curve $D \subseteq \mathbf{P}^{6}$ of genus 22 containing an elliptic curve $E \subseteq \mathbf{P}^{6}$ with $\operatorname{deg}(E)=7$ as a component such that the union of $D$ and some point $p \in \mathbf{P}^{6} \backslash E$ is the syzygy scheme of a rank 6 linear syzygy among quadrics in $\mathbf{P}^{6}$.

Furthermore, given a reducible spin curve $D=C \cup_{e} E \subseteq \mathbf{P}^{6}$ of genus 22 as above, that is, with $\omega_{D} \cong \mathcal{O}_{D}(2)$, the genus 8 component $C$ has a nontrivial syzygy of rank 6 involving the quadrics in the 6-dimensional subspace $I_{D}(2) \subseteq I_{C}(2)$, see Lemma 29 for a proof of this fact.

\section{B. Syzygies and quartics singular along paracanonical curves}

We first discuss an alternative characterization of the non-surjectivity of the map $\mu_{C, L}$ :

Proposition 5. Assume the paracanonical curve $\phi_{L}(C)$ is projectively normal and scheme-theoretically cut out by quadrics. Then $K_{1,2}(C, L) \neq 0$ if and only if there exists a degree 4 homogeneous polynomial on $\boldsymbol{P}^{6}$, which vanishes to order at least 2 along $C$ but does not belong to the image of the multiplication map $\operatorname{Sym}^{2} I_{C, L}(2) \rightarrow I_{C, L}(4)$.

Proof. We work on the variety $X \stackrel{\tau}{\rightarrow} \mathbf{P}^{6}$ defined as the blow-up of $\mathbf{P}^{6}$ along $\phi_{L}(C)$. Let $E$ be the exceptional divisor of the blow-up, and consider the line bundle $H:=\tau^{*} \mathcal{O}_{\mathbf{P}^{6}}(2)(-E)$ on $X$. Its space of sections identifies to $I_{C}(2)$, and our assumption that $C$ is scheme-theoretically cut out by quadrics says equivalently that $H$ is a globally generated line bundle on $X$. The nonvanishing of $K_{1,2}(C, L)$ is equivalent to the non-surjectivity of the multiplication map

$$
H^{0}(X, H) \otimes H^{0}\left(X, \tau^{*} \mathcal{O}(1)\right) \rightarrow H^{0}\left(X, H \otimes \tau^{*} \mathcal{O}(1)\right),
$$

where we use the identification

$$
H^{0}\left(X, H \otimes \tau^{*} \mathcal{O}(1)\right)=H^{0}\left(X, \tau^{*} \mathcal{O}(3)(-E)\right)=I_{C}(3) .
$$


As $H$ is globally generated by its space $W:=I_{C}(2)$ of global sections, the Koszul complex

$$
0 \rightarrow \bigwedge^{7} W \otimes \mathcal{O}_{X}(-7 H) \rightarrow \ldots \rightarrow \bigwedge^{2} W \otimes \mathcal{O}_{X}(-2 H) \rightarrow W \otimes \mathcal{O}_{X}(-H) \rightarrow \mathcal{O}_{X} \rightarrow 0
$$

is exact. We now twist this complex by $\tau^{*} \mathcal{O}_{\mathbf{P}^{6}}(1)(H)$ and take global sections. The last map is then the multiplication map (2.4). The successive terms of this twisted complex are

$$
\bigwedge^{i} W \otimes \mathcal{O}_{X}\left(\tau^{*} \mathcal{O}(1)\right)((-i+1) H)
$$

for $0 \leq i \leq 7$. The spectral sequence abutting to the hypercohomology of this complex, that is 0 , has

$$
E_{2}^{0,0}=\operatorname{coker}\left\{W \otimes H^{0}\left(X, \tau^{*} \mathcal{O}(1)\right) \rightarrow H^{0}\left(X, H \otimes \tau^{*} \mathcal{O}(1)\right)\right\}
$$

and the terms $E_{1}^{i,-i-1}$ for $i<-1$ are equal to $\bigwedge^{-i} W \otimes H^{-i-1}\left(X, \tau^{*} \mathcal{O}(1)((i+1) H)\right)$. Similarly, we have

$$
E_{1}^{i,-i}=\bigwedge^{-i} W \otimes H^{-i}\left(X, \tau^{*} \mathcal{O}(1)((i+1) H)\right)
$$

Lemma 6. (i) We have

$$
E_{1}^{i,-i-1}=\bigwedge^{-i} W \otimes H^{-i-1}\left(X, \tau^{*} \mathcal{O}(1)((i+1) H)\right)=0
$$

for $-i-1=5, \ldots, 1$.

(ii) For $-i-1=6$, that is, $i=-7$, we have

$$
E_{1}^{-7,6}=\bigwedge^{7} W \otimes H^{6}\left(X, \tau^{*} \mathcal{O}(1)(-6 H)\right)=\bigwedge^{7} W \otimes I_{C}(4)_{2}^{\vee},
$$

where $I_{C}(4)_{2} \subseteq I_{C}(4)$ is the set of quartic polynomials vanishing at order at least 2 along $C$, and

$$
E_{1}^{-6,6}=\bigwedge^{6} W \otimes H^{6}\left(X, \tau^{*} \mathcal{O}(1)(-5 H)\right)=\bigwedge^{6} W \otimes I_{C}(2)^{\vee}
$$

(iii) We have $E_{1}^{i,-i}=0$, for $-6<i<0$.

Proof of Lemma 6. (i) We want equivalently to show that

$$
H^{\ell}\left(X, \tau^{*} \mathcal{O}(1)(-\ell H)\right)=0, \text { when } \ell=5, \ldots, 1 .
$$

Recall that $H=\tau^{*} \mathcal{O}(2)(-E)$. Furthermore,

$$
K_{X}=\tau^{*} \mathcal{O}_{\mathbf{P}^{6}}(-7)(4 E)
$$

So we have to prove that

$$
H^{\ell}\left(X, \tau^{*} \mathcal{O}(-2 \ell+1)(\ell E)\right)=0, \text { for } \ell=5, \ldots, 1 .
$$

Examining the spectral sequence induced by $\tau$, and using the fact that

$$
R^{s} \tau_{*}\left(\mathcal{O}_{X}(t E)\right)=0
$$


for $s \neq 0,4$ and also for $s=4, t \leq 4$, we see that for $1 \leq \ell \leq 4$,

$$
H^{\ell}\left(X, \tau^{*} \mathcal{O}(-2 \ell+1)(\ell E)\right)=H^{\ell}\left(\mathbf{P}^{6}, \mathcal{O}(-2 \ell+1) \otimes R^{0} \tau_{*} \mathcal{O}_{X}(\ell E)\right)
$$

For $1 \leq \ell \leq 4$, the right hand side is zero, because it is equal to $H^{\ell}\left(\mathbf{P}^{6}, \mathcal{O}(-2 \ell+1)\right)$.

For $\ell=5$, we have to compute the space $H^{5}\left(X, \tau^{*} \mathcal{O}(-9)(5 E)\right)$, which by Serre duality and by (2.10), is dual to the space

$$
H^{1}\left(X, \tau^{*} \mathcal{O}(2)(-E)\right)=H^{1}\left(\mathbf{P}^{6}, \mathcal{O}(2) \otimes \mathcal{I}_{C}\right)=0 .
$$

(ii) We have to compute the spaces $H^{6}\left(X, \tau^{*} \mathcal{O}(1)(-6 H)\right)$ and $H^{6}\left(X, \tau^{*} \mathcal{O}(1)(-5 H)\right)$. As $H:=$ $\tau^{*} \mathcal{O}(2)(-E)$, this is rewritten as $H^{6}\left(X, \tau^{*} \mathcal{O}(-11)(6 E)\right)$ and $H^{6}\left(X, \tau^{*} \mathcal{O}(-9)(5 E)\right)$ respectively. If we dualize using (2.10), we get

$$
\begin{gathered}
H^{6}\left(X, \tau^{*} \mathcal{O}(-11)(6 E)\right)^{\vee}=H^{0}\left(X, \tau^{*} \mathcal{O}(4)(-2 E)\right)=I_{C}(4)_{2}, \\
H^{6}\left(X, \tau^{*} \mathcal{O}(-9)(5 E)\right)^{\vee}=H^{0}\left(X, \tau^{*} \mathcal{O}(2)(-E)\right)=I_{C}(2) .
\end{gathered}
$$

(iii) We have

$$
E_{1}^{i,-i}=E_{1}^{-6,6}=\bigwedge^{-i} W \otimes H^{-i}\left(X, \tau^{*} \mathcal{O}(1)((i+1) H)\right)=\bigwedge^{-i} W \otimes H^{-i}\left(X, \tau^{*} \mathcal{O}(2 i+3)((-i-1) E)\right) .
$$

For $1 \leq-i \leq 5$, we have $R^{s} \tau_{*} \mathcal{O}_{X}((-i-1) E)=0$ unless $s=0$. Furthermore, we have

$$
R^{0} \tau_{*} \mathcal{O}_{X}((-i-1) E)=\mathcal{O}_{\mathbf{P}^{6}}
$$

so that

$$
H^{-i}\left(X, \tau^{*} \mathcal{O}(2 i+3)((-i-1) E)\right)=H^{-i}\left(\mathbf{P}^{6}, \mathcal{O}_{\mathbf{P}^{6}}(2 i+3)\right)=0
$$

Corollary 7. Only one $E_{2}^{p, q}$-terms of this spectral sequence is possibly nonzero in degree -1, namely

$$
E_{2}^{-7,6}=\operatorname{Ker}\left\{\bigwedge^{7} W \otimes I_{C}(4)_{2}^{\vee} \rightarrow \bigwedge^{6} W \otimes I_{C}(2)^{\vee}\right\}
$$

Furthermore, all the differentials $d_{r}$ starting from $E_{2}^{-7,6}$ vanish for $2 \leq r<7$.

Note that the map

$$
\bigwedge^{7} W \otimes I_{C}(4)_{2}^{\vee} \rightarrow \bigwedge^{6} W \otimes I_{C}(2)^{\vee}
$$

is nothing but the transpose of the multiplication map

$$
W \otimes I_{C}(2) \rightarrow I_{C}(4)_{2},
$$

up to trivialization of $\bigwedge^{7} W$. It follows that

$$
\left(E_{2}^{-7,6}\right)^{\vee}=\operatorname{Coker}\left\{W \otimes I_{C}(2) \rightarrow I_{C}(4)_{2}\right\} .
$$

Corollary 7 concludes the proof of the proposition since it implies that we have an isomorphism given by $d_{7}$ between (2.12) and (2.6), or a perfect duality between (2.12) and the cokernel (2.13).

Proposition 5 has the following consequence. Recall that $P_{8}^{14}$ is the moduli space of pairs $[C, L]$, with $C$ being a smooth curve of genus 8 and $L \neq K_{C}$ a paracanonical line bundle. 
Proposition 8. The Koszul divisor $\mathfrak{K} 0 \mathfrak{s z}$ of $P_{8}^{14}$ is the union of two divisors, one of them being the set of pairs $[C, L]$ such that $\phi_{L}(C)$ is not scheme-theoretically cut out by quadrics, the other being the set of pairs $[C, L]$ such that $H^{1}\left(\boldsymbol{P}^{6}, \mathcal{I}_{C}^{2}(4)\right) \neq 0$, or equivalently, such that there exists a quartic which is singular along $\phi_{L}(C)$ but does not lie in $\operatorname{Sym}^{2} I_{C}(2)$.

Proof. We first have to prove that the locus of pairs $[C, L]$ such that $\phi_{L}(C)$ is not scheme-theoretically cut-out by quadrics is contained in the divisor $\mathfrak{K} \mathfrak{o} \mathfrak{s}$. This is a consequence of the following lemmas:

Lemma 9. If $L \neq K_{C}$ is a projectively normal paracanonical line bundle on a curve of genus 8 , then $\phi_{L}(C)$ is scheme-theoretically cut out by cubics.

Proof of Lemma 9. We observe that the twisted ideal sheaf $\mathcal{I}_{C}(3)$ is regular in Castelnuovo-Mumford sense. Indeed, we have

$$
H^{i}\left(\mathbf{P}^{6}, \mathcal{I}_{C}(3-i)\right)=H^{i-1}\left(C, L^{\otimes(3-i)}\right)
$$

for $i \geq 2$, and the right hand side is obviously 0 for $i-1 \geq 2$, and also 0 for $i-1=1$ since $H^{1}(C, L)=0$ because $L \neq K_{C}$ and $\operatorname{deg} L=2 g-2$. For $i=1$, we have

$$
H^{1}\left(\mathbf{P}^{6}, \mathcal{I}_{C}(2)\right)=0
$$

by projective normality. Being regular, the sheaf $\mathcal{I}_{C}(3)$ is generated by global sections.

Corollary 10. If $C, L$ are as above, and $C$ is not scheme-theoretically cut out by quadrics, then the multiplication map

$$
I_{C}(2) \otimes H^{0}\left(\boldsymbol{P}^{6}, \mathcal{O}_{P^{6}}(1)\right) \rightarrow I_{C}(3)
$$

is not surjective.

To conclude the proof of the proposition, we just have to show that the sublocus of $P_{8}^{14}$ where $L$ is not projectively normal is not a divisor, since the statement of the proposition will be then an immediate consequence of Proposition 5. We argue along the lines of [12]. First of all, a line bundle $L$ of degree 14 is not generated by sections if and only if $L=K_{C}(-x+y)$ for some points $x, y \in C$. This determines a codimension 6 locus of $P_{8}^{14}$. Similarly $L$ is not very ample if and only if $L=K_{C}(-x-y+z+t)$, for some points $x, y, z, t$ of $C$, which is satisfied in a codimension 4 locus of $P_{8}^{14}$. Finally, assume $L$ is very ample but $\phi_{L}(C)$ is not projectively normal. Equivalently

$$
\operatorname{Sym}^{2} H^{0}(C, L) \rightarrow H^{0}\left(C, L^{\otimes 2}\right)
$$

is not surjective, which means that there exists a rank 2 vector bundle $F$ on $C$ which is a nontrivial extension

$$
0 \longrightarrow K_{C} \otimes L^{\vee} \longrightarrow F \longrightarrow L \longrightarrow 0
$$

such that $h^{0}(C, F)=7$. If $x, y, z \in C$, there exists a nonzero section $\sigma \in H^{0}(C, F)$ vanishing on $x, y$ and $z$, and thus $F$ is also an extension

$$
0 \longrightarrow D \longrightarrow F \longrightarrow K_{C} \otimes D^{\vee} \longrightarrow 0,
$$

where $D$ is a line bundle such that $h^{0}(C, D(-x-y-z)) \neq 0$, and $h^{0}\left(C, L \otimes D^{\vee}\right) \neq 0$. We thus have $h^{0}(C, D)+h^{0}\left(C, K_{C} \otimes D^{\vee}\right) \geq 7$ and $\operatorname{Cliff}(D) \leq 2$. As $D$ is effective of degree at least 3 , one has the following possibilities:

a) $h^{0}\left(C, K_{C} \otimes D^{\vee}\right)=0$, and then $D=L$, which contradicts the fact that the extension (2.14) is not split;

b) $h^{0}\left(C, K_{C} \otimes D^{\vee}\right)=1$ and $h^{0}(C, D) \geq 6$, and then $D=L(-x)$ and $h^{0}\left(K_{C} \otimes L^{\vee}(x)\right) \neq 0$, so $L=K_{C}(x-y)$, which happens in a locus of codimension at least 6 in $P_{8}^{14}$;

c) $D$ contributes to the Clifford index of $C$. As the locus of curves $[C] \in \mathcal{M}_{8}$ with $\operatorname{Cliff}(C) \leq 2$ is of codimension 2 in $\mathcal{M}_{8}$, this situation does not occur in codimension 1. 
We shall need later on the following result:

Lemma 11. Let $\phi_{L}: C \hookrightarrow \boldsymbol{P}^{6}$ be a projectively normal paracanonical curve of genus 8 . If $C$ is scheme-theoretically cut out by quadrics, the multiplication map

$$
\mathrm{Sym}^{2} I_{C, L}(2) \rightarrow I_{C, L}(4)
$$

is injective.

Proof. As the restriction map $\phi_{L}^{*}: H^{0}\left(\mathbf{P}^{6}, \mathcal{O}_{\mathbf{P}^{6}}(2)\right) \rightarrow H^{0}\left(C, L^{\otimes 2}\right)$ is surjective, its kernel $I_{C, L}(2)$ is of dimension 7. Let as before $\tau: X \rightarrow \mathbf{P}^{6}$ be the blow-up of $\mathbf{P}^{6}$ along $\phi_{L}(C)$, and let $E$ be its exceptional divisor. We view $I_{C, L}(2)$ as $H^{0}\left(X, \tau^{*} \mathcal{O}(2)(-E)\right)$ and our assumption is that $I_{C, L}(2)$ generates the line bundle $H:=\tau^{*} \mathcal{O}(2)(-E)$ everywhere on $X$. Thus $I_{C, L}(2)$ provides a morphism

$$
\psi: X \rightarrow \mathbf{P}\left(I_{C, L}(2)\right)
$$

Now we have $\operatorname{deg} c_{1}(H)^{6} \neq 0$ by Sublemma 12 below, and thus the morphism $\psi$ has to be generically finite, hence dominant since both spaces have dimension 6 . It is then clear that the pull-back map

$$
\psi^{*}: H^{0}\left(\mathbf{P}\left(I_{C, L}(2)\right), \mathcal{O}(2)\right) \rightarrow H^{0}\left(X, H^{\otimes 2}\right)
$$

is injective. On the other hand, this morphism is nothing but the map (2.15).

Sublemma 12. With the same notation as above, we have

$$
\operatorname{deg} c_{1}(H)^{6}=8
$$

Proof. We have

$$
c_{1}(H)^{6}=\sum_{i}\left(\begin{array}{l}
6 \\
i
\end{array}\right)(-2)^{i} h^{i} \cdot E^{6-i}
$$

where $h:=\tau^{*} c_{1}\left(\mathcal{O}_{\mathbf{P}^{6}}(1)\right)$, and

$$
h^{i} \cdot E^{6-i}=0
$$

for $i \neq 6,1,0$. Furthermore

$$
h^{6}=1, \text { and } h \cdot E^{5}=\operatorname{deg} \phi_{L}(C)=14
$$

and $E^{6}=c_{1}\left(N_{C}\right)$. By adjunction formula

$$
\operatorname{deg} c_{1}\left(N_{C}\right)=7 \operatorname{deg} \phi_{L}(C)+\operatorname{deg} K_{C}=8 \cdot 14 .
$$

It follows that

$$
\operatorname{deg} c_{1}(H)^{6}=64-6 \cdot 28+8 \cdot 14=8
$$

which proves (2.17).

Proposition 5 and Lemma 11 describe precisely the splitting of the Koszul divisor $\mathfrak{K} \mathfrak{o s z}$ into the divisors $\mathfrak{K}_{0} \mathfrak{s}_{6}$ and $\mathfrak{K}_{0} \mathfrak{s}_{7}$ corresponding to paracanonical curves $[C, L] \in P_{8}^{14}$ having a non-zero syzygy $\gamma \in K_{1,2}(C, L)$ of rank 6 or respectively 7. Precisely, $\mathfrak{K}_{\mathfrak{o g} \mathfrak{z}_{6}}$ is a unirational divisor (cf. [3] Theorem 5.3) consisting of those paracanonical curves $C \subseteq \mathbf{P}^{6}$ for which $H^{1}\left(\mathbf{P}^{6}, \mathcal{I}_{C}^{2}(4)\right) \neq 0$. The divisor $\mathfrak{K}_{\mathfrak{i} \mathfrak{z} 7}$ consists of paracanonical curves $C \subseteq \mathbf{P}^{6}$ which are not scheme-theoretically cut out by quadrics. 


\section{First proof: reducible spin curves}

\section{A. The syzygy is degenerate}

The first observation is the following result (already observed experimentally in [3]), which turns out to be useful for the description given below of the general paracanonical curve of genus 8 with nontrivial syzygies.

Proposition 13. Let $C \subseteq P^{6}$ be a smooth paracanonical curve of genus 8 and degree 14, schemetheoretically generated by quadrics. Then a nontrivial syzygy

$$
\gamma \in \operatorname{Ker}\left\{I_{C}(2) \otimes H^{0}\left(\mathcal{O}_{P^{6}}(1)\right) \rightarrow I_{C}(3)\right\}
$$

must be degenerate, that is of rank at most 6 .

Proof. We use the morphism

$$
\psi: X \rightarrow \mathbf{P}\left(I_{C}(2)\right)
$$

introduced in (2.16), where $\tau: X \rightarrow \mathbf{P}^{6}$ is the blow-up of $C$ with exceptional divisor $E$, and $H:=$ $\tau^{*} \mathcal{O}_{\mathbf{P}^{6}}(-2 E)$. This gives us a morphism

$$
(\tau, \psi): X \rightarrow \mathbf{P}^{6} \times \mathbf{P}^{6}
$$

which is of degree 1 on its image, and the syzygy $\gamma$ induces a hypersurface $Y$ of bidegree $(1,1)$ in $\mathbf{P}^{6} \times \mathbf{P}^{6}$ containing the 6 -dimensional variety $(\tau, \psi)(X)$. Assume to the contrary that $\gamma$ has maximal rank 7, or equivalently that $Y$ is smooth. Then by the Lefschetz Hyperplane Restriction Theorem, the restriction map $H^{10}\left(\mathbf{P}^{6} \times \mathbf{P}^{6}, \mathbb{Z}\right) \rightarrow H^{10}(Y, \mathbb{Z})$ is surjective, so that $[(\tau, \psi)(X)]_{Y} \in H^{10}(Y, \mathbb{Z})$ is the restriction of a class $\beta \in H^{10}\left(\mathbf{P}^{6} \times \mathbf{P}^{6}, \mathbb{Z}\right)$, which implies that

$$
[(\tau, \psi)(X)]=\beta \cdot[Y] \text { in } H^{12}\left(\mathbf{P}^{6} \times \mathbf{P}^{6}, \mathbb{Z}\right),
$$

where $[Y] \in H^{2}\left(\mathbf{P}^{6} \times \mathbf{P}^{6}, \mathbb{Z}\right)$ is the class of $Y$, that is $h_{1}+h_{2}$, with $h_{i}$ for $i=1,2$ being the pull-backs of the hyperplane classes on each factor. Note that $H^{12}\left(\mathbf{P}^{6} \times \mathbf{P}^{6}, \mathbb{Z}\right)$ is the set of degree 6 homogeneous polynomials with integral coefficients in $h_{1}$ and $h_{2}$. We now have:

Lemma 14. An element $\alpha \in H^{12}\left(\boldsymbol{P}^{6} \times \boldsymbol{P}^{6}, \mathbb{Z}\right)$ is of the form $\left(h_{1}+h_{2}\right) \cdot \beta$ if and only if it satisfies the condition

$$
\sum_{i=0}^{6}(-1)^{i} h_{1}^{i} \cdot h_{2}^{6-i} \cdot \alpha=0 \text { in } H^{24}\left(\boldsymbol{P}^{6} \times \boldsymbol{P}^{6}, \mathbb{Z}\right)=\mathbb{Z} .
$$

Proof of Lemma 14. We have $\left(h_{1}+h_{2}\right) \cdot\left(\sum_{i}(-1)^{i} h_{1}^{i} \cdot h_{2}^{6-i}\right)=0$ in $H^{14}\left(\mathbf{P}^{6} \times \mathbf{P}^{6}, \mathbb{Z}\right)$, so one implication is obvious. That the two conditions are equivalent then follows from the fact that both conditions determine a saturated corank 1 sublattice of $H^{12}\left(\mathbf{P}^{6} \times \mathbf{P}^{6}, \mathbb{Z}\right)$.

To conclude that $\gamma$ has to be degenerate, in view of Lemma 14, it suffices to prove that the class $[(\tau, \psi)(X)]$ does not satisfy (3.19). Since $(\tau, \psi)^{*} h_{1}=c_{1}(H)$ and $(\tau, \psi)^{*} h_{2}=2 c_{1}(H)-E$, it is enough to prove that

$$
\sum_{i=0}^{6}(-1)^{i} c_{1}(H)^{i} \cdot\left(2 c_{1}(H)-E\right)^{6-i} \neq 0
$$

which follows from the computations made in the proof of Sublemma 12. 


\section{B. Syzygies and spin curves of genus 22 in $\mathrm{P}^{6}$}

Recall that $\overline{\mathcal{S}}_{g}^{-}$denotes the moduli stack of odd stable spin curves of genus $g$, see [5] for details. We start with a nodal genus 22 spin curve of the form $[D:=C \cup E, \vartheta] \in \overline{\mathcal{S}}_{22}^{-}$, where $C$ is a smooth genus 8 curve, $E$ is a smooth elliptic curve and $e:=C \cap E$ consists of 14 distinct points, thus $p_{a}(D)=22$. Assume $\vartheta \in \operatorname{Pic}^{21}(D)$ verifies $\vartheta^{\otimes 2} \cong \omega_{D}$, hence the restricted line bundles $\vartheta_{E}$ and $\vartheta_{C}$ have degrees 7 and 14 respectively. Furthermore, $h^{0}\left(E, \vartheta_{E}\right)=7$, whereas $h^{0}\left(C, \vartheta_{C}\right)=7$ if and only if $\vartheta_{C} \nsucceq K_{C}$. The intersection divisor $e$ on the two components of $D$ is characterized by

$$
e_{C} \in\left|\vartheta_{C}^{\otimes 2} \otimes K_{C}^{\vee}\right| \text { and } e_{E} \in\left|\vartheta_{E}^{\otimes 2}\right| \text {. }
$$

Note in particular that $e_{C} \in\left|K_{C}\right|$ if and only if $\vartheta_{C}^{\otimes 2}=K_{C}^{\otimes 2}$, that is $\left(C, \vartheta_{C}\right)$ is canonical or Prym canonical.

The line bundle $\vartheta$ on $D$ fits into the Mayer-Vietoris exact sequence:

$$
0 \longrightarrow \vartheta \longrightarrow \vartheta_{C} \oplus \vartheta_{E} \stackrel{r}{\longrightarrow} \mathcal{O}_{e}(\vartheta) \longrightarrow 0
$$

where $r$ is defined by the isomorphisms on the fibers of $\vartheta_{C}$ and $\vartheta_{E}$ over the points in $e$. Given $\vartheta_{C} \in \operatorname{Pic}^{14}(C)$ with $\vartheta_{C}^{\otimes 2}=K_{C}(e)$ and $\vartheta_{E} \in \operatorname{Pic}^{7}(E)$ with $\vartheta_{E}^{\otimes 2}=\mathcal{O}_{E}(e)$, there is a finite number of stable spin curves $[D, \theta] \in \overline{\mathcal{S}}_{22}^{-}$such that the restrictions of $\vartheta$ to $C$ and $E$ are isomorphic to $\vartheta_{C}$ and $\vartheta_{E}$ respectively. Passing to global sections in the Mayer-Vietoris sequence, we obtain the exact sequence:

$$
0 \longrightarrow H^{0}(D, \vartheta) \longrightarrow H^{0}\left(C, \vartheta_{C}\right) \oplus H^{0}\left(E, \vartheta_{E}\right) \stackrel{r}{\longrightarrow} H^{0}\left(\mathcal{O}_{e}(\vartheta)\right) \longrightarrow \cdots
$$

Note that $r$ is represented by a $14 \times 14$ matrix and $h^{0}(D, \vartheta)=14-\operatorname{rk}(r)$. In the case of a reducible spin curve coming from the syzygy of a paracanonical genus 8 curve in $\mathfrak{K}_{0} \mathfrak{s z}_{6}$, one has $h^{0}(D, \vartheta)=\operatorname{rk}(r)=7$.

\section{C. Proof of Theorem 1 via reducible spin curves}

Theorem 1 states that every Prym canonical curve of genus 8 has a syzygy of rank 6 . First we observe the existence of such a curve having the generic behavior described in Lemma 3 .

Lemma 15. There exists a curve $[C, \eta] \in \mathcal{R}_{8}$, whose Prym canonical model is scheme theoretically cut out by quadrics, and $K_{2,1}\left(C, K_{C} \otimes \eta\right)$ is 1-dimensional, generated by a syzygy $\gamma$ of rank 6 . The syzygy scheme of $\gamma$ is the union of a point $p$ and a nodal curve $D=C \cup E$, such that $E$ is a smooth elliptic curve of degree 7 and $e:=C \cdot E \in\left|K_{C}\right|$ consists of 14 mutually distinct points. Moreover, no cubic polynomial on $\boldsymbol{P}^{6}$ vanishes with multiplicity 2 along $C$.

Proof. Examples of singular Prym canonical curves having all these properties have been produced in [3] Proposition 4.4 or [4]. A generic deformation in $\overline{\mathcal{R}}_{8}$ of these singular examples will provide the required smooth Prym canonical curve.

(First) proof of Theorem 1 . We denote by $X$ the moduli space of elements $\left[C, \eta, x_{1}, \ldots, x_{14}\right]$, where $[C, \eta] \in \mathcal{R}_{8}$ is a smooth Prym curve of genus 8 and $x_{i} \in C$ are pairwise distinct points such that $x_{1}+\cdots+x_{14} \in\left|K_{C}\right| \cong \mathbf{P}^{7}$. Since the fibres of the forgetful map $X \rightarrow \mathcal{R}_{8}$ are 7-dimensional, it follows that $X$ is an irreducible variety of dimension 28 .

Let $T$ be the locally closed parameter space of odd genus 22 spin curves having the form

$$
\left(\left[D:=C \cup_{\left\{x_{1}, \ldots, x_{14}\right\}} E, \vartheta\right]:[C] \in \mathcal{M}_{8}, \sum_{i=1}^{14} x_{i} \in\left|K_{C}\right|,\left[E, x_{1}, \ldots, x_{14}\right] \in \mathcal{M}_{1,14}, \vartheta^{\otimes 2}=\omega_{D}\right) .
$$


Observe that points in $T$, apart from the spin structure $[D, \vartheta] \in \overline{\mathcal{S}}_{22}^{-}$also carry an underlying Prym structure $\left[C, \eta:=K_{C} \otimes \vartheta_{C}^{\vee}\right] \in \mathcal{R}_{8}$, for $\vartheta_{C}^{\otimes 2} \cong K_{C}\left(x_{1}+\cdots+x_{14}\right) \cong K_{C}^{\otimes 2}$. One has an induced finite morphism $T \rightarrow X \times \mathcal{M}_{1,14}$, as well as a map $\mu: T \rightarrow \mathcal{R}_{8}$ forgetting the 14-pointed elliptic curve. It follows that $\operatorname{dim} T=\operatorname{dim} X+\operatorname{dim} \mathcal{M}_{1,14}=42$. The locus

$$
T_{7}:=\left\{[D, \vartheta] \in T: h^{0}(D, \vartheta) \geq 7\right\}
$$

has the structure of a skew-symmetric degeneracy locus. Applying [13] Theorem 1.10, each component of $T_{7}$ has codimension at most $\left(\begin{array}{l}7 \\ 2\end{array}\right)=21$ inside $T$, that is, $\operatorname{dim}\left(T_{7}\right) \geq \operatorname{dim}\left(\mathcal{R}_{8}\right)$.

By passing to a general 8-nodal Prym canonical curve [C, $\eta]$, following [3] Proposition 4.5, as well as Lemma 15, we have that $\operatorname{dim} K_{1,2}\left(C, K_{C} \otimes \eta\right)=1$. In particular, the fibre $\mu^{-1}([C, \eta])$ contains an isolated point, which shows that $T_{7}$ is non-empty and has a component which maps dominantly under $\mu$ onto $\mathcal{R}_{8}$. Theorem 1 now follows.

Remark 16. The same construction can be carried out at the level of general paracanonical curves $[C, L] \in P_{8}^{14}$, where $L \in \operatorname{Pic}^{14}(C) \backslash\left\{K_{C}\right\}$. The key difference is that we replace $T$ by a variety $T^{\prime}$ parametrizing objects

$$
\begin{gathered}
\left(\left[D:=C \cup_{\left\{x_{1}, \ldots, x_{14}\right\}} E, \vartheta, L\right]:\left[C, x_{1}, \ldots, x_{14}\right] \in \mathcal{M}_{14,8}, L \in \operatorname{Pic}^{14}(C) \backslash\left\{K_{C}\right\},\right. \\
\left.\sum_{i=1}^{14} x_{i} \in\left|L^{\otimes 2} \otimes K_{C}^{\vee}\right|,\left[E, x_{1}, \ldots, x_{14}\right] \in \mathcal{M}_{1,14}, \vartheta^{\otimes 2}=\omega_{D}\right) .
\end{gathered}
$$

Similarly, we have a morphism $\mu^{\prime}: T^{\prime} \rightarrow P_{8}^{14}$ retaining the pair $[C, L]$ alone. The main difference compared to the Prym canonical case is that now

$$
\operatorname{dim}\left|L^{\otimes 2} \otimes K_{C}^{\vee}\right|=6,
$$

therefore $\operatorname{dim}\left(T^{\prime}\right)=\operatorname{dim}\left(P_{8}^{14}\right)+\operatorname{dim}\left(\mathcal{M}_{1,14}\right)+6=49$. The degeneracy locus $T_{7}^{\prime} \subseteq T^{\prime}$ defined by the condition $\left.h^{0}(D, \vartheta) \geq 7\right)$ has codimension 21 inside $T^{\prime}$, that is,

$$
\operatorname{dim}\left(T_{7}^{\prime}\right)=28=\operatorname{dim}\left(P_{8}^{14}\right)-1
$$

It follows that the image $\mu^{\prime}\left(T_{7}^{\prime}\right) \subseteq P_{8}^{14}$ has codimension 1 , which is in accordance with $\mathfrak{K} 0 \mathfrak{s z}_{6}$ being a divisor in $P_{8}^{14}$.

\section{Second proof: Divisor class calculations on $\overline{\mathcal{R}}_{g}$}

Recall [10] that $\overline{\mathcal{R}}_{g}$ is the Deligne-Mumford moduli space of Prym curves of genus $g$, whose geometric points are triples $[X, \eta, \beta]$, where $X$ is a quasi-stable curve of genus $g, \eta \in \operatorname{Pic}(X)$ is a line bundle of total degree 0 such that $\eta_{E}=\mathcal{O}_{E}(1)$ for each smooth rational component $E \subseteq X$ with $|E \cap \overline{X-E}|=2$ (such a component is said to be exceptional), and $\beta: \eta^{\otimes 2} \rightarrow \mathcal{O}_{X}$ is a sheaf homomorphism whose restriction to any non-exceptional component is an isomorphism. If $\pi: \overline{\mathcal{R}}_{g} \rightarrow \overline{\mathcal{M}}_{g}$ is the map dropping the Prym structure, one has the formula

$$
\pi^{*}\left(\delta_{0}\right)=\delta_{0}^{\prime}+\delta_{0}^{\prime \prime}+2 \delta_{0}^{\mathrm{ram}} \in C H^{1}\left(\overline{\mathcal{R}}_{g}\right),
$$

where $\delta_{0}^{\prime}:=\left[\Delta_{0}^{\prime}\right], \delta_{0}^{\prime \prime}:=\left[\Delta_{0}^{\prime \prime}\right]$, and $\delta_{0}^{\text {ram }}:=\left[\Delta_{0}^{\text {ram }}\right]$ are irreducible boundary divisor classes on $\overline{\mathcal{R}}_{g}$, which we describe by specifying their respective general points.

We choose a general point $\left[C_{x y}\right] \in \Delta_{0} \subset \overline{\mathcal{M}}_{g}$ corresponding to a smooth 2-pointed curve $(C, x, y)$ of genus $g-1$ and consider the normalization map $\nu: C \rightarrow C_{x y}$, where $\nu(x)=\nu(y)$. A general point 
of $\Delta_{0}^{\prime}$ (respectively of $\Delta_{0}^{\prime \prime}$ ) corresponds to a pair $\left[C_{x y}, \eta\right]$, where $\eta \in \operatorname{Pic}^{0}\left(C_{x y}\right)[2]$ and $\nu^{*}(\eta) \in \operatorname{Pic}^{0}(C)$ is non-trivial (respectively, $\nu^{*}(\eta)=\mathcal{O}_{C}$ ). A general point of $\Delta_{0}^{\text {ram }}$ is a Prym curve of the form $(X, \eta)$, where $X:=C \cup_{\{x, y\}} \mathbf{P}^{1}$ is a quasi-stable curve with $p_{a}(X)=g$ and $\eta \in \operatorname{Pic}^{0}(X)$ is a line bundle such that $\eta_{\mathbf{P}^{1}}=\mathcal{O}_{\mathbf{P}^{1}}(1)$ and $\eta_{C}^{2}=\mathcal{O}_{C}(-x-y)$. In this case, the choice of the homomorphism $\beta$ is uniquely determined by $X$ and $\eta$. In what follows, we work on the partial compactification $\widetilde{\mathcal{R}}_{g} \subseteq \overline{\mathcal{R}}_{g}$ of $\mathcal{R}_{g}$ obtained by removing the boundary components $\pi^{-1}\left(\Delta_{j}\right)$ for $j=1, \ldots,\left\lfloor\frac{g}{2}\right\rfloor$, as well as $\Delta_{0}^{\prime \prime}$. In particular, $C H^{1}\left(\widetilde{\mathcal{R}}_{g}\right)=\mathbb{Q}\left\langle\lambda, \delta_{0}^{\prime}, \delta_{0}^{\mathrm{ram}}\right\rangle$.

For a stable Prym curve $[X, \eta] \in \widetilde{\mathcal{R}}_{g}$, set $L:=\omega_{X} \otimes \eta \in \operatorname{Pic}^{2 g-2}(X)$ to be the paracanonical bundle. For $i \geq 1$, we introduce the vector bundle $\mathcal{N}_{k}$ over $\widetilde{\mathcal{R}}_{g}$, having fibres

$$
\mathcal{N}_{k}[X, \eta]=H^{0}\left(X, L^{\otimes k}\right) .
$$

The first Chern class of $\mathcal{N}_{k}$ is computed in [10] Proposition 1.7:

$$
c_{1}\left(\mathcal{N}_{k}\right)=\left(\begin{array}{l}
k \\
2
\end{array}\right)\left(12 \lambda-\delta_{0}^{\prime}-2 \delta_{0}^{\mathrm{ram}}\right)+\lambda-\frac{k^{2}}{4} \delta_{0}^{\mathrm{ram}} .
$$

Then we define the locally free sheaves $\mathcal{G}_{k}$ on $\widetilde{\mathcal{R}}_{g}$ via the exact sequences

$$
0 \longrightarrow \mathcal{G}_{k} \longrightarrow \operatorname{Sym}^{k} \mathcal{N}_{1} \longrightarrow \mathcal{N}_{k} \longrightarrow 0,
$$

that is, satisfying $\mathcal{G}_{k}[X, \eta]:=I_{X, L}(k) \subseteq \operatorname{Sym}^{k} H^{0}(X, L)$. Using (4.22) one computes $c_{1}\left(\mathcal{G}_{k}\right)$.

We also need the class of the vector bundle $\mathcal{G}$ with fibres

$$
\mathcal{G}[X, \eta]=H^{0}\left(X, \omega_{X}^{\otimes 5} \otimes \eta^{\otimes 4}\right)=H^{0}\left(X, \omega_{X} \otimes L^{\otimes 4}\right) .
$$

Lemma 17. One has $c_{1}(\mathcal{G})=121 \lambda-10 \delta_{0}^{\prime}-24 \delta_{0}^{\mathrm{ram}} \in C H^{1}\left(\widetilde{\mathcal{R}}_{g}\right)$.

Proof. We apply Grothendieck-Riemann-Roch to the universal Prym curve $f: \mathcal{C} \rightarrow \widetilde{\mathcal{R}}_{g}$. Denote by $\mathcal{L} \in \operatorname{Pic}(\mathcal{C})$ the universal Prym bundle, whose restriction to each Prym curve is the corresponding 2 -torsion point, that is, $\mathcal{L}_{\mid f^{-1}([X, \eta])}=\eta$, for each point $[X, \eta] \in \widetilde{\mathcal{R}}_{g}$. Since $R^{1} f_{*}\left(\omega_{f}^{\otimes 5} \otimes \mathcal{L}^{\otimes 4}\right)=0$, we write

$$
c_{1}(\mathcal{G})=f_{*}\left[\left(1+5 c_{1}\left(\omega_{f}\right)+4 c_{1}(\mathcal{L})+\frac{\left(5 c_{1}\left(\omega_{f}\right)+4 c_{1}(\mathcal{L})\right)^{2}}{2}\right) \cdot\left(1-\frac{c_{1}\left(\omega_{f}\right)}{2}+\frac{c_{1}^{2}\left(\Omega_{f}^{1}\right)+[\operatorname{Sing}(f)]}{12}\right)\right]_{2} .
$$

We use then the formulas $f_{*}\left(c_{1}^{2}(\mathcal{L})\right)=-\delta_{0}^{\text {ram }} / 2$ and $f_{*}\left(c_{1}(\mathcal{L}) \cdot c_{1}\left(\omega_{f}\right)\right)=0$ (see [10], Proposition 1.6) coupled with Mumford's formula $f_{*}\left(c_{1}^{2}\left(\Omega_{f}^{1}\right)+[\operatorname{Sing}(f)]\right)=12 \lambda$ as well with the identity

$$
\kappa_{1}:=f_{*}\left(c_{1}^{2}\left(\omega_{f}\right)\right)=12 \lambda-\delta_{0}^{\prime}-2 \delta_{0}^{\mathrm{ram}},
$$

in order to conclude.

The Koszul locus

$$
\mathcal{Z}_{8}:=\mathfrak{K} \mathfrak{o s z} \cap \mathcal{R}_{8}=\left\{[C, \eta] \in \mathcal{R}_{8}: K_{1,2}\left(C, K_{C} \otimes \eta\right) \neq 0\right\}
$$

is a virtual divisor on $\mathcal{R}_{8}$, that is, the degeneracy locus of a map between vector bundles of the same rank over $\widetilde{\mathcal{R}}_{8}$. If it is a genuine divisor (which we aim to rule out), the class of its closure in $\widetilde{\mathcal{R}}_{8}$ is given by [3] Theorem F:

$$
\left[\overline{\mathcal{Z}}_{8}\right]=27 \lambda-4 \delta_{0}^{\prime}-6 \delta_{0}^{\mathrm{ram}} \in C H^{1}\left(\widetilde{\mathcal{R}}_{8}\right) .
$$


Remark 18. Some of the considerations above can be extended to higher order torsion points. We recall that $\mathcal{R}_{g, \ell}$ is the moduli space of pairs $[C, \eta]$, where $C$ is a smooth curve of genus $g$ and $\eta \in \operatorname{Pic}^{0}(C)$ is a non-trivial $\ell$-torsion point. It is then shown in [3] that the locus $\mathcal{Z}_{8, \ell}:=\mathfrak{K} \mathfrak{o s z} \cap \mathcal{R}_{8, \ell} \subseteq P_{8}^{14}$ is a divisor on $\mathcal{R}_{8, \ell}$ for each other level $\ell \geq 3$. The class of the compactification of $\mathcal{Z}_{8, \ell}$ is given by the following formula, see [3] Theorem F:

$$
\left[\overline{\mathcal{Z}}_{8, \ell}\right]=27 \lambda-4 \delta_{0}^{\prime}-\sum_{a=1}^{\left\lfloor\frac{\ell}{2}\right\rfloor} \frac{4\left(a^{2}-a \ell+\ell^{2}\right)}{\ell} \delta_{0}^{(a)} \in C H^{1}\left(\widetilde{\mathcal{R}}_{8, \ell}\right) .
$$

We refer to [3] Section 1.4, for the definition of the boundary divisor classes $\delta_{0}^{(a)}$, where $a=1, \ldots,\left\lfloor\frac{\ell}{2}\right\rfloor$. If $\pi: \overline{\mathcal{R}}_{g, \ell} \rightarrow \overline{\mathcal{M}}_{g}$ is the map forgetting the level $\ell$ structure, then

$$
\pi^{*}\left(\delta_{0}\right)=\delta_{0}^{\prime}+\delta_{0}^{\prime \prime}+\ell \sum_{\ell=1}^{\left\lfloor\frac{\ell}{2}\right\rfloor} \delta_{0}^{(a)} .
$$

We fix now a genus 8 Prym-canonically embedded curve $\phi_{L}: C \hookrightarrow \mathbf{P}^{6}$. As usual, we denote the kernel bundle by $M_{L}:=\Omega_{\mathbf{P}^{6} \mid C}^{1}(1)$, hence we have the exact sequence

$$
0 \longrightarrow N_{C}^{\vee} \otimes L^{\otimes 4} \longrightarrow M_{L} \otimes L^{\otimes 3} \longrightarrow K_{C} \otimes L^{\otimes 4} \longrightarrow 0 .
$$

This can be interpreted as an exact sequence of vector bundles over $\widetilde{\mathcal{R}}_{8}$. Denoting by $\mathcal{H}$ the vector bundle over $\widetilde{\mathcal{R}}_{8}$ with fibres $H^{0}\left(C, N_{C}^{\vee} \otimes L^{\otimes 4}\right)$, we compute using the previous formulas and the fact that $\operatorname{rk}\left(\mathcal{N}_{1}\right)=h^{0}(C, L)=7$ and $\operatorname{rk}\left(\mathcal{N}_{3}\right)=h^{0}\left(C, L^{\otimes 3}\right)=35$ :

$$
c_{1}(\mathcal{H})=35 c_{1}\left(\mathcal{N}_{1}\right)+7 c_{1}\left(\mathcal{N}_{3}\right)-c_{1}\left(\mathcal{N}_{4}\right)-c_{1}(\mathcal{G})=100 \lambda-5 \delta_{0}^{\prime}-\frac{53}{2} \delta_{0}^{\mathrm{ram}} .
$$

Thus $\mathfrak{D}_{1}=\mathfrak{K}_{\mathfrak{L} \mathfrak{s}_{7}} \cap \mathcal{R}_{8}$ and $\mathfrak{D}_{2}=\mathfrak{K}_{\mathfrak{n} \mathfrak{s}_{6}} \cap \mathcal{R}_{8}$. We have already seen in Proposition 5 that $K_{1,2}(C, L) \neq 0$ if and only if either $\phi_{L}(C) \subseteq \mathbf{P}^{6}$ is not scheme-theoretically cut out by quadrics, or else, $H^{1}\left(\mathbf{P}^{6}, \mathcal{I}_{C}^{2}(4)\right) \neq 0$. We write

$$
\mathcal{Z}_{8}=\mathfrak{D}_{1}+\mathfrak{D}_{2}, \quad \text { where }
$$

$$
\mathfrak{D}_{1}:=\left\{[C, \eta] \in \mathcal{R}_{8}: \phi_{L}(C) \subseteq \mathbf{P}^{6} \text { is scheme-theoretically not cut out by quadrics }\right\}
$$

and

$$
\mathfrak{D}_{2}:=\left\{[C, \eta] \in \mathcal{R}_{8}: H^{1}\left(\mathbf{P}^{6}, \mathcal{I}_{C}^{2}(4)\right) \neq 0\right\} .
$$

We have already observed that $\operatorname{dim} I_{C, L}(2)=7$ and $\chi\left(\mathbf{P}^{6}, \mathcal{I}_{C}^{2}(4)\right)=28$. If $\mathcal{Z}_{8}$ is a divisor, then $\mathfrak{D}_{2}$ is a divisor as well and for $[C, \eta] \in \mathcal{R}_{8} \backslash \mathfrak{D}_{2}$, we have that

$$
\operatorname{dim} \operatorname{Sym}^{2} I_{C, L}(2)=\operatorname{dim} I_{C, L}(4)_{2}=28 .
$$

Paying some attention to its definition, the divisor $\mathfrak{D}_{1}$ can be thought as the degeneracy locus

$$
\left\{[C, \eta] \in \mathcal{R}_{8}: \operatorname{Sym}^{2} I_{C, L}(2) \stackrel{\neq}{\longrightarrow} I_{C, L}(4)_{2}\right\},
$$

which is an effective divisor on $\widetilde{\mathcal{R}}_{8}$. We compute the class of this divisor:

Theorem 19. We have the following formulas:

$$
\left[\overline{\mathfrak{D}}_{1}\right]=7 \lambda-\frac{1}{2} \delta_{0}^{\prime}-\frac{3}{4} \delta_{0}^{\mathrm{ram}} \in C H^{1}\left(\widetilde{\mathcal{R}}_{8}\right)
$$

and

$$
\left[\overline{\mathfrak{D}}_{2}\right]=20 \lambda-\frac{7}{2} \delta_{0}^{\prime}-\frac{21}{4} \delta_{0}^{\mathrm{ram}} \in C H^{1}\left(\widetilde{\mathcal{R}}_{8}\right) .
$$


Proof. We first globalize over $\widetilde{\mathcal{R}}_{8}$ the following exact sequence:

$$
0 \longrightarrow I_{C, L}(4)_{2} \longrightarrow I_{C, L}(4) \longrightarrow H^{0}\left(C, N_{C}^{\vee} \otimes L^{\otimes 4}\right) \longrightarrow H^{1}\left(\mathbf{P}^{6}, \mathcal{I}_{C}^{2}(4)\right) \longrightarrow 0 .
$$

Denote by $\mathcal{A}$ the sheaf on $\widetilde{\mathcal{R}}_{8}$ supported along the divisor $\mathfrak{D}_{2}$, whose fibre over a general point of that divisor is equal to to $H^{1}\left(\mathbf{P}^{6}, \mathcal{I}_{C}^{2}(4)\right)$. There is a surjective morphism of sheaves

$$
\mathcal{H} \rightarrow \mathcal{A}
$$

and denote by $\mathcal{G}_{4}^{\prime}$ its kernel. Since $\mathcal{A}$ is locally free along $\mathfrak{D}_{2}$ and $\widetilde{\mathcal{R}}_{8}$ is a smooth stack, using the Auslander-Buchsbaum formula we find that $\mathcal{G}_{4}^{\prime}$ is a locally free sheaf of rank equal to $\operatorname{rk}(\mathcal{H})=$ $\chi\left(C, N_{C}^{\vee}(4 L)\right)=19 \cdot 7$. Precisely, $\mathcal{G}_{4}^{\prime}$ is an elementary transformation of $\mathcal{H}$ along the divisor $\mathfrak{D}_{2}$. Furthermore, $c_{1}\left(\mathcal{G}_{4}^{\prime}\right)=c_{1}(\mathcal{H})-\left[\overline{\mathfrak{D}}_{2}\right]$.

The morphism $\mathcal{G}_{4} \rightarrow \mathcal{H}$ globalizing the maps $I_{C, L}(4) \rightarrow H^{0}\left(C, N_{C}^{\vee} \otimes L^{\otimes 4}\right)$ factors through the subsheaf $\mathcal{G}_{4}^{\prime}$ and we form the exact sequence:

$$
0 \longrightarrow \mathcal{G}_{4}^{2} \longrightarrow \mathcal{G}_{4} \longrightarrow \mathcal{G}_{4}^{\prime} \longrightarrow 0
$$

The multiplication maps $\operatorname{Sym}^{2} I_{C, L}(2) \rightarrow I_{C, L}(4)_{2}$ globalize to a sheaf morphism

$$
\nu: \operatorname{Sym}^{2}\left(\mathcal{G}_{2}\right) \rightarrow \mathcal{G}_{4}^{2}
$$

between locally free sheaves of the same rank 28 over the stack $\widetilde{\mathcal{R}}_{8}$. The degeneration locus of $\nu$ is precisely the divisor $\overline{\mathfrak{D}}_{1}$. We compute:

$$
c_{1}\left(\operatorname{Sym}^{2}\left(\mathcal{G}_{2}\right)\right)=8 c_{1}\left(\mathcal{G}_{2}\right)=8\left(8 c_{1}\left(\mathcal{N}_{1}\right)-c_{1}\left(\mathcal{N}_{2}\right)\right)=-40 \lambda+8\left(\delta_{0}^{\prime}+\delta_{0}^{\mathrm{ram}}\right),
$$

and

$$
c_{1}\left(\mathcal{G}_{4}^{2}\right)=120 c_{1}\left(\mathcal{N}_{1}\right)-c_{1}\left(\mathcal{N}_{4}\right)-c_{1}(\mathcal{H})+\left[\overline{\mathfrak{D}}_{2}\right]=-53 \lambda+11 \delta_{0}^{\prime}+\frac{25}{2} \delta_{0}^{\mathrm{ram}}+\left[\overline{\mathfrak{D}}_{2}\right] .
$$

We obtain the relation $\left[\overline{\mathfrak{D}_{1}}\right]-\left[\overline{\mathfrak{D}}_{2}\right]=-13 \lambda+3 \delta_{0}^{\prime}+\frac{9}{2} \delta_{0}^{\text {ram }}$. Since at the same time

$$
\left[\overline{\mathfrak{D}}_{1}\right]+\left[\overline{\mathfrak{D}}_{2}\right]=\left[\mathcal{Z}_{8}\right]=27 \lambda-4 \delta_{0}^{\prime}-6 \delta_{0}^{\mathrm{ram}},
$$

we solve the system and conclude.

We are now in a position to give a second proof of Theorem 1:

Theorem 20. The class $\left[\overline{\mathfrak{D}}_{2}\right]$ cannot be effective. It follows that $\mathcal{Z}_{8}=\mathcal{R}_{8}$ and $K_{1,2}\left(C, K_{C} \otimes \eta\right) \neq 0$, for every Prym curve $[C, \eta] \in \mathcal{R}_{8}$.

Proof. We use the sweeping curve of the boundary divisor $\Delta_{0}^{\prime}$ of $\widetilde{\mathcal{R}}_{8}$ constructed via Nikulin surfaces in [11] Lemma 3.2: Precisely, through the general point of $\Delta_{0}^{\prime}$ there passes a rational curve $\Gamma \subseteq \Delta_{0}^{\prime}$, entirely contained in $\widetilde{\mathcal{R}}_{8}$, having the following numerical characters:

$$
\Gamma \cdot \lambda=8, \quad \Gamma \cdot \delta_{0}^{\prime}=42, \quad \text { and } \quad \Gamma \cdot \delta_{0}^{\mathrm{ram}}=8 .
$$

We note that $\Gamma \cdot \overline{\mathfrak{D}}_{2}<0$. Writing $\overline{\mathcal{D}}_{2} \equiv \alpha \cdot \delta_{0}^{\prime}+E$, where $\alpha \geq 0$ and $E$ is an effective divisor whose support is disjoint from $\Delta_{0}^{\prime}$, we immediately obtain a contradiction. 
The divisors $\mathfrak{D}_{1}$ and $\mathfrak{D}_{2}$ can be defined in an identical manner at the level of each moduli space $\overline{\mathcal{R}}_{8, \ell}$ of twisted level $\ell$ curves of genus $g$. As already pointed out, in the case $\ell \geq 3$ it follows from [3] Proposition 4.4 that both $\mathfrak{D}_{1}$ and $\mathfrak{D}_{2}$ are actual divisors. Repeating the same calculations as for $\ell=2$, we obtain the following formula on the partial compactification $\widetilde{\mathcal{R}}_{8, \ell}$ of $\mathcal{R}_{8, \ell}$ :

$$
\left[\overline{\mathfrak{D}}_{2}\right]=20 \lambda-\frac{7}{2} \delta_{0}^{\prime}-\sum_{a=1}^{\left\lfloor\frac{\ell}{2}\right\rfloor} \frac{1}{2 \ell}\left(7 a^{2}-7 a \ell+17 \ell^{2}-20 \ell\right) \delta_{0}^{(a)} \in C H^{1}\left(\widetilde{\mathcal{R}}_{8, \ell}\right) .
$$

As an application, we mention a different proof of one of the main results from [1]:

Theorem 21. The canonical class of $\overline{\mathcal{R}}_{8, \ell}$ is big for $\ell \geq 3$. It follows that $\overline{\mathcal{R}}_{8, \ell}$ is a variety of general type for $\ell=3,4,6$.

Proof. Using formula (4.25), it is a routine exercise to check that for $\ell \geq 3$ the canonical class computed in [3] Proposition 1.5

$$
K_{\widetilde{\mathcal{R}}_{8, \ell}}=13 \lambda-2 \delta_{0}^{\prime}-(\ell+1) \sum_{a=1}^{\left\lfloor\frac{\ell}{2}\right\rfloor} \delta_{0}^{(a)}
$$

can be written as a positive combination of the big class $\lambda$ and the effective class $\left[\overline{\mathfrak{D}}_{2}\right]$, hence it is big. Arguing along the lines of [3] Remark 3.5, it is easy to extend this result to the full compactification $\overline{\mathcal{R}}_{8, \ell}$ and deduce that $K_{\overline{\mathcal{R}}_{8, \ell}}$ is big.

To conclude that $\overline{\mathcal{R}}_{8, \ell}$ is of general type, one needs, apart from the bigness of the canonical class $K_{\widetilde{\mathcal{R}}_{8, \ell}}$ of the moduli stack, a result that the singularities of the coarse moduli space $\overline{\mathcal{R}}_{8, \ell}$ impose no adjunction conditions. This is only known for $2 \leq \ell \leq 6, \ell \neq 5$, see [2].

\section{Rank 2 vector bundles and singular quartics}

Our goal in this section is to propose a construction of syzygies of Prym canonical curves of genus 8 . We also sketch the proof of the fact that these syzygies are nontrivial. We fix again a general element $[C, \eta] \in \mathcal{R}_{8}$ and set $L:=K_{C} \otimes \eta$. According to Proposition 5, in order to prove that $K_{2,1}(C, L) \neq 0$, we have to produce quartic hypersurfaces in $\mathbf{P}^{6}$ which vanish at order at least 2 along $\phi_{L}(C)$, but do not lie in the image of the map $\operatorname{Sym}^{2} I_{C, L}(2) \rightarrow I_{C, L}(4)$. The goal of this section is to produce such quartics from rank 2 vector bundles on $C$. The (incomplete) proof that the quartics we construct are not in the image of $\operatorname{Sym}^{2} I_{C, L}(2)$ depends on an unproved general position statement $(*)$, but there might be other approaches exploiting the fact that the hypersurfaces in question are determinantal.

The following construction produces quartics vanishing at order 2 along $C$. Let $E$ be a rank 2 vector bundle on $C$, with determinant $K_{C}$. Assume

$$
h^{0}(C, E)=4, \quad h^{0}(C, E(\eta))=4 .
$$

Setting $V_{0}:=H^{0}(C, E)$ and $V_{1}:=H^{0}(C, E(\eta))$, we have a natural map

$$
V_{0} \otimes V_{1} \rightarrow H^{0}(C, L),
$$

defined using evaluation and the following composite map:

$$
H^{0}(E) \otimes H^{0}(E(\eta)) \rightarrow H^{0}(E \otimes E(\eta)) \cong H^{0}(\mathcal{E} n d E \otimes L) \stackrel{\operatorname{Tr}}{\longrightarrow} H^{0}(C, L) .
$$

This map gives dually a morphism

$$
H^{0}(C, L)^{\vee} \rightarrow V_{0}^{\vee} \otimes V_{1}^{\vee},
$$

(which will be proved below to be injective for a general choice of $E$ ). We consider the quartic hypersurface $D_{4}$ on $\mathbf{P}\left(V_{0}^{\vee} \otimes V_{1}^{\vee}\right)$ parametrizing tensors of rank at most 3 . 
Lemma 22. The restriction $D_{4, E}$ of this quartic to $\boldsymbol{P}\left(H^{0}(C, L)^{\vee}\right) \subseteq \boldsymbol{P}\left(V_{0}^{\vee} \otimes V_{1}^{\vee}\right)$ is singular along the curve $C$.

Proof. The quartic $D_{4}$ is singular along the set $T_{2} \subseteq \mathbf{P}\left(V_{0}^{\vee} \otimes V_{1}^{\vee}\right)$ of tensors of rank at most 2. The quartic $D_{4, E}$ in $\mathbf{P}\left(H^{0}(C, L)^{\vee}\right)$ is thus singular along $T_{2} \cap \mathbf{P}\left(H^{0}(C, L)^{\vee}\right)$, which obviously contains $C \subseteq \mathbf{P}\left(H^{0}(C, L)^{\vee}\right)$, since at a point $p \in C$, the map $V_{0} \otimes V_{1} \rightarrow H^{0}(C, L)$ composed with the evaluation at $p$ factors through $E_{\mid p} \otimes E(\eta)_{\mid p}$.

By Brill-Noether theory, the variety $W_{7}^{1}(C)$ of degree 7 pencils on $C$ is 4 -dimensional. There should thus exist finitely many elements $D \in W_{7}^{1}(C)$ with the property that

$$
h^{0}(C, D) \geq 2, h^{0}(C, D \otimes \eta) \geq 2 .
$$

We now have the following lemma:

Lemma 23. Let $[C, \eta] \in \mathcal{R}_{8}$ be as above and $D \in W_{7}^{1}(C)$ satisfying (5.28). Then

(i) $h^{0}(C, D)=2$ and $h^{0}(C, D \otimes \eta)=2$. The multiplication map

$$
\left(H^{0}(C, D) \otimes H^{0}\left(C, K_{C} \otimes D^{\vee}\right)\right) \oplus\left(H^{0}(C, D \otimes \eta) \otimes H^{0}\left(C, K_{C} \otimes D^{\vee} \otimes \eta\right)\right) \rightarrow H^{0}\left(C, K_{C}\right)
$$

is surjective (in fact, an isomorphism).

(ii) The multiplication map

$$
\left(H^{0}(C, D) \otimes H^{0}\left(C, K_{C} \otimes D^{\vee} \otimes \eta\right)\right) \oplus\left(H^{0}(C, D \otimes \eta) \otimes H^{0}\left(C, K_{C} \otimes D^{\vee}\right)\right) \rightarrow H^{0}\left(C, K_{C}(\eta)\right)
$$

is surjective.

Proof. This can be proved by a degeneration argument, for example by degenerating $C$ to the union of two curves of genus 4 meeting at one point.

By Brill-Noether theory, the following corollary follows from (i) above:

Corollary 24. For $[C, \eta]$ as above, the set of pencils $D \in W_{7}^{1}(C)$ satisfying (5.28) is finite.

Given such a $D$, we form the rank 2 vector bundle

$$
E=D \oplus\left(K_{C} \otimes D^{\vee}\right)
$$

on $C$ which satisfies the conditions (5.26). The associated quartic is however not interesting for our purpose, due to the following fact:

Lemma 25. The quartic on $\boldsymbol{P}\left(H^{0}(C, L)^{\vee}\right)$ associated to the vector bundle $D \oplus\left(K_{C} \otimes D^{\vee}\right)$ is the union of the two quadrics $Q_{0}$ and $Q_{1}$ associated respectively with the multiplication maps

$$
H^{0}(D) \otimes H^{0}\left(\left(K_{C} \otimes D^{\vee}\right)(\eta)\right) \rightarrow H^{0}\left(K_{C}(\eta)\right) \text { and } H^{0}(D(\eta)) \otimes H^{0}\left(K_{C} \otimes D^{\vee}\right) \rightarrow H^{0}\left(K_{C}(\eta)\right) .
$$

Both these quadrics contain $C$.

Proof. Indeed we have in this case

$$
\begin{gathered}
V_{0}=H^{0}(C, E)=H^{0}(C, D) \oplus H^{0}\left(C, K_{C} \otimes D^{\vee}\right), \text { respectively } \\
V_{1}=H^{0}(C, E(\eta))=H^{0}(C, D \otimes \eta) \oplus H^{0}\left(C, K_{C} \otimes D^{\vee} \otimes \eta\right) .
\end{gathered}
$$


Furthermore, it is clear that the map of (5.27) factors through the projection

$$
V_{0} \otimes V_{1} \rightarrow\left(H^{0}(C, D) \otimes H^{0}\left(C, K_{C} \otimes D^{\vee} \otimes \eta\right)\right) \oplus\left(H^{0}\left(C, K_{C} \otimes D^{\vee}\right) \otimes H^{0}(C, D \otimes \eta)\right)
$$

and induces on each summand the multiplication map. The quadric $Q_{0}$ is by definition associated with the the multiplication map

$$
\mu_{0}: H^{0}(C, D) \otimes H^{0}\left(C, K_{C} \otimes D^{\vee} \otimes \eta\right) \rightarrow H^{0}\left(C, K_{C} \otimes \eta\right),
$$

and is the set of elements $f$ in $\mathbf{P}\left(H^{0}\left(K_{C} \otimes \eta\right)\right)^{\vee}$ such that $\mu_{0}^{*}(f)$ is a tensor of rank $\leq 1$. Similarly for $Q_{1}$, with $D$ being replaced with $D(\eta)$. Finally we use the fact that a tensor

$$
\left(\mu_{0}^{*} f, \mu_{1}^{*} f\right) \in\left(H^{0}(C, D) \otimes H^{0}\left(C, K_{C} \otimes D^{\vee} \otimes \eta\right)\right) \oplus\left(H^{0}\left(C, K_{C} \otimes D^{\vee}\right) \otimes H^{0}(C, D \otimes \eta)\right)
$$

has rank at most 3 if and only if one of $\mu_{0}^{*} f$ and $\mu_{1}^{*} f$ has rank at most 1 .

We recall from [15] or [14] that the Brill-Noether condition $h^{0}(C, E) \geq 4$ imposes only $10=\left(\begin{array}{l}5 \\ 2\end{array}\right)$ equations on the parameter space of rank 2 vector bundles $E$ with determinant $K_{C}$. As det $E(\eta) \cong K_{C}$ as well, we conclude that the equations (5.26) impose only 20 conditions. As the moduli space $\mathcal{S U}_{C}\left(2, K_{C}\right)$ of semistable rank 2 vector bundles on $C$ having determinant $K_{C}$ has dimension $3 g-3=$ 21 , in our case we conclude that there is a positive dimensional family of such vector bundles on $C$ satisfying (5.26).

We now sketch the proof of the fact that for $C$ general of genus 8 and $D \in W_{7}^{1}(C)$ satisfying (5.28), for a general deformation $E$ of the vector bundle $D \oplus\left(K_{C} \otimes D^{\vee}\right)$ satisfying $\operatorname{det} E \cong K_{C}$ and $h^{0}(C, E)=4$, the associated quartic $D_{4, E}$ singular along $C$ is not defined by an element of $\operatorname{Sym}^{2} I_{C}(2)$. Combined with Proposition 5, this provides a third approach to Theorem 1. The proof of this fact rests on an unproven general position statement $(*)$, so it is incomplete.

Sketch of proof of the nontriviality of the syzygy. The vector bundle $E$ is generated by sections, as it is a general section-preserving deformation of the vector bundle

$$
D \oplus\left(K_{C} \otimes D^{\vee}\right)
$$

which is generated by global sections, and similarly for $E(\eta)$. Along $C \subseteq \mathbf{P}\left(H^{0}(C, L)^{\vee}\right)$, then the rational map

$$
\mathbf{P}\left(H^{0}(C, L)^{\vee}\right) \rightarrow \mathbf{P}\left(H^{0}(E)^{\vee} \otimes H^{0}(E(\eta))^{\vee}\right)
$$

is well-defined and the image of $C$ is contained in the locus $T_{2, E}$ of tensors of rank exactly 2 . In fact, the case of $D \oplus\left(K_{C} \otimes D^{\vee}\right)$ shows that this map is a morphism for general $E$ (one just needs to know that $H^{0}\left(C, K_{C} \otimes \eta\right)$ is generated by the two vector spaces $H^{0}(D) \otimes H^{0}\left(K_{C} \otimes D^{\vee} \otimes \eta\right)$ and $H^{0}(D \otimes \eta) \otimes H^{0}\left(K_{C} \otimes D^{\vee}\right)$ respectively, or rather their images under the multiplication map. Note that on $T_{2, E}$, there is a rank 2 vector bundle $M$ which restricts to $E$ on $C$.

In the case of the split vector bundle $E_{\mathrm{sp}}=D \oplus\left(K_{C} \otimes D^{\vee}\right)$, Lemma 25 shows that the Zariski closure $\overline{T_{2, E_{\mathrm{sp}}}}$ parameterizing tensors of rank $\leq 2$ in $\mathbf{P}\left(H^{0}(C, L)^{\vee}\right) \subseteq \mathbf{P}\left(V_{0}^{\vee} \otimes V_{1}^{\vee}\right)$ is equal to the singular locus of $D_{4, E_{\mathrm{sp}}}$ and consists of the union of the two planes $P_{0}, P_{1}$ defined as the singular loci of the quadrics $Q_{0}, Q_{1}$ respectively, and the intersection $Q_{0} \cap Q_{1}$. The locus $\overline{T_{2, E_{\mathrm{sp}}}} \backslash T_{2, E_{\mathrm{sp}}}$ is the locus where the tensor has rank 1, and this happens exactly along the two conics $P_{0} \cap Q_{1}$ and $P_{1} \cap Q_{0}$. The curve $C$ is contained in $Q_{0} \cap Q_{1}$ and does not intersect $P_{0} \cup P_{1}$. In particular, the rational map $\phi: \widetilde{\mathbf{P}^{6}} \rightarrow \mathbf{P}^{6}$ given by the linear system $I_{C}(2)$ is well defined along $P_{0} \cup P_{1}$. We believe that the following general position statement concerning the two planes $P_{i}$ is true for general $C$ and $D, \eta$ as above. 
(*) The surfaces $\phi\left(P_{i}\right)$ are projectively normal Veronese surfaces, generating a hyperplane $\left\langle\phi\left(P_{i}\right)\right\rangle \subseteq$ $\boldsymbol{P}^{6}$. Furthermore, the surface $\phi\left(P_{0}\right) \cup \phi\left(P_{1}\right) \subseteq \boldsymbol{P}^{6}$ is contained in a unique quadric in $\boldsymbol{P}^{6}$, namely the union of the two hyperplanes $\left\langle\phi\left(P_{0}\right)\right\rangle$ and $\left\langle\phi\left(P_{1}\right)\right\rangle$.

We now prove that, assuming $(*)$, for a general vector bundle $E$ as above, the associated quartic $D_{4, E}$ singular along $C$ is not defined by an element of $\operatorname{Sym}^{2} I_{C}(2)$. As $P_{0}, P_{1}$ are 2-dimensional reduced components of $\overline{T_{2, E_{\mathrm{sp}}}}$, hence of the right dimension, the theory of determinantal hypersurfaces shows that for general $E$ as above, there is a reduced surface $\Sigma_{E} \subseteq \overline{T_{2, E}}$ whose specialization when $E=E_{\mathrm{sp}}$ contains $P_{0} \cup P_{1}$. Let $\mathcal{E} \rightarrow C \times B$ be a family of vector bundles on $C$ parameterized by a smooth curve $B$, with general fiber $E$ and special fiber $E_{\mathrm{sp}}$. Denote by $\mathcal{E}_{b}$ the restriction of $\mathcal{E}$ to $C \times\{b\}$. Property $(*)$ then implies that $\phi\left(\Sigma_{\mathcal{E}_{b}}\right)$ for general $b \in B$ is contained in at most one quadric $Q_{\mathcal{E}_{b}}$ in $\mathbf{P}^{6}$. We argue by contradiction and assume that the quartic $D_{4, \mathcal{E}_{b}}$ is a pull-back $\phi^{-1}(Q)$ for general $b$. One thus must have $Q=Q_{\mathcal{E}_{b}}$. Next, the determinantal quartic $D_{4, \mathcal{E}_{b}}$ is singular along $T_{2, \mathcal{E}_{b}}$, hence along $\Sigma_{\mathcal{E}_{b}}$. Let $b \mapsto q_{\mathcal{E}_{b}} \in \operatorname{Sym}^{2} I_{C}(2)$, where $q_{\mathcal{E}_{b}}$ is a defining equation for the quadric $Q_{\mathcal{E}_{b}}$. Then we find that the first order derivative of the family $\phi^{*} q_{\mathcal{E}_{b}}$ at $b_{0}$ also vanishes along $\Sigma_{\mathcal{E}_{b_{0}}}$, hence it must be proportional to $\phi^{*} q_{\mathcal{E}_{b_{0}}}$. We then conclude that the quadric $Q_{\mathcal{E}_{b}}$ is in fact constant, and thus must be equal to the quadric $Q_{E_{\mathrm{sp}}}$. We now reach a contradiction by proving the following lemma.

Lemma 26. If the determinantal quartic $D_{4, \mathcal{E}_{b}}$ is constant, equal to $D_{\mathrm{sp}}=Q_{0} \cup Q_{1}$, then the vector bundle $\mathcal{E}_{b}$ on $C$ does not deform with $b \in B$.

Proof. Denoting $V_{0, b}:=H^{0}\left(C, \mathcal{E}_{b}\right), V_{1, b}:=H^{0}\left(C, \mathcal{E}_{b}(\eta)\right)$, we have the multiplication map

$$
V_{0, b} \otimes V_{1, b} \rightarrow H^{0}\left(C, K_{C} \otimes \eta\right)
$$

which is surjective for generic $b$ since it is surjective for $\mathcal{E}_{0}=D \oplus\left(K_{C} \otimes D^{\vee}\right)$ (see Lemma 23). The determinantal quartic $D_{4, \mathcal{E}_{b}}$ is the vanishing locus of the determinant of the corresponding bundle map

$$
\sigma_{b}: V_{0, b} \otimes \mathcal{O}_{\mathbf{P}\left(H^{0}\left(C, K_{C}(\eta)\right)^{\vee}\right)} \rightarrow V_{1, b}^{\vee} \otimes \mathcal{O}_{\mathbf{P}\left(H^{0}\left(C, K_{C}(\eta)\right)^{\vee}\right)}(1)
$$

on $\mathbf{P}\left(H^{0}\left(C, K_{C} \otimes \eta\right)^{\vee}\right)$. We know that $D_{4, \mathcal{E}_{b}}=Q_{0} \cup Q_{1}$ for any $b \in B$, where the quadrics $Q_{i}$ are singular (of rank 4), but with singular locus $P_{i}$ not intersecting $C \subseteq Q_{0} \cap Q_{1}$. The morphism $\sigma_{b}$ has rank exactly 1 generically along each $Q_{i}$ and the kernel of $\sigma_{\mid D_{4, b}}$ determines a line bundle $\mathcal{K}_{i, b}$ on its smooth locus $Q_{i} \backslash P_{i}$. This line bundle is independent of $b$ since $\operatorname{Pic}\left(Q_{i} \backslash P_{i}\right)$ has no continuous part. The restriction of $\mathcal{K}_{i, b}$ to $C$ is thus constant. Finally, on the smooth part of $\left(Q_{0} \cap Q_{1}\right)_{\text {reg }}$, the kernel $\operatorname{Ker}(\sigma)$ contains the two line bundles $\mathcal{K}_{i, b \mid Q_{0} \cap Q_{1}}$. Restricting to $C \subseteq\left(Q_{0} \cap Q_{1}\right)_{\text {reg }}$, we conclude that $\operatorname{Ker} \sigma_{b \mid C}$ contains $\mathcal{K}_{i, 0 \mid C}$ for $i=0,1$. For $b=0$, one has

$$
\operatorname{Ker} \sigma_{0 \mid C}=\mathcal{K}_{0,0 \mid C} \oplus \mathcal{K}_{1,0 \mid C}
$$

and this thus remains true for general $b$. Finally, it follows from the construction and the fact that $\mathcal{E}_{b}$ is generated by its sections that $\operatorname{Ker} \sigma_{b \mid C}=\mathcal{E}_{b}^{\vee}$, which finishes the proof.

\section{Miscellany}

\section{A. Extra remarks on the geometry of paracanonical curves of genus 8 with a nontrivial syzygy}

We now comment on an interesting rank 2 vector bundle appearing in our situation. Again, let $\phi_{L}: C \hookrightarrow \mathbf{P}^{6}$ be a paracanonical curve of genus 8 . We assume $L$ is scheme-theoretically cut out by quadrics. Denoting by $N_{C}$ the normal bundle of $C$ in the embedding in $\mathbf{P}^{6}$, we consider the natural 
map $I_{C}(2) \otimes \mathcal{O}_{C} \rightarrow N_{C}^{\vee} \otimes L^{\otimes 2}$ (which is surjective by our assumption) given by differentiation along $\phi_{L}(C)$, and let $F$ denote its kernel. We thus have the short exact sequence:

$$
0 \longrightarrow F \longrightarrow I_{C}(2) \otimes \mathcal{O}_{C} \longrightarrow N_{C}^{\vee} \otimes L^{\otimes 2} \longrightarrow 0 .
$$

If $K_{1,2}(C, L) \neq 0$, the map $\mu: I_{C}(2) \otimes H^{0}\left(\mathbf{P}^{6}, \mathcal{O}(1)\right) \rightarrow I_{C}(3)$ is not surjective, hence not injective. A fortiori, the map

$$
\bar{\mu}: I_{C}(2) \otimes H^{0}\left(\mathbf{P}^{6}, \mathcal{O}_{\mathbf{P}^{6}}(1)\right) \rightarrow H^{0}\left(C, N_{C}^{\vee} \otimes L^{\otimes 3}\right)
$$

induced by $(6.30)$ is not injective, so that $h^{0}(C, F(L)) \neq 0$. In fact, the equivalence between the statements $h^{0}(C, F(L)) \neq 0$ and $K_{1,2}(C, L) \neq 0$ follows from the same argument once we know that there is no cubic polynomial on $\mathbf{P}^{6}$ vanishing with multiplicity 2 along $C$.

We observe now that $F$ is a vector bundle of rank 2 on the curve $C$, with determinant equal to $\operatorname{det} N_{C} \otimes L^{\otimes(-2)} \cong K_{C} \otimes L^{\otimes(-3)}$. Hence if $F(L)$ has a nonzero section, assuming this section vanishes nowhere along $C$, then $F(L)$ is an extension of $K_{C} \otimes L^{\vee}$ by $\mathcal{O}_{C}$. This provides an extension class

$$
e \in H^{1}\left(C, L \otimes K_{C}^{\vee}\right)=H^{0}\left(C, K_{C}^{\otimes 2} \otimes L^{\vee}\right)^{\vee} .
$$

Assume now $L \otimes K_{C}^{\vee}=: \eta$ is a nonzero 2-torsion element of $\operatorname{Pic}^{0}(C)$. Then

$$
e \in H^{0}(C, L)^{\vee} \text {. }
$$

On the other hand, according to Theorem 20, there exists a nontrivial syzygy

$$
\gamma=\sum_{i=1}^{6} \ell_{i} \otimes q_{i} \in K_{1,2}(C, L)=\operatorname{Ker}\left\{H^{0}\left(\mathbf{P}^{6}, \mathcal{O}_{\mathbf{P}^{6}}(1)\right) \otimes I_{C}(2) \rightarrow I_{C}(3)\right\},
$$

which is degenerate by Proposition 13. As we saw already, it has in fact rank 6 for generic $[C, \eta]$, hence determines a nonzero element

$$
f \in H^{0}\left(\mathbf{P}^{6}, \mathcal{O}_{\mathbf{P}^{6}}(1)\right)^{\vee}=H^{0}(C, L)^{\vee}=H^{1}\left(C, K_{C} \otimes L^{\vee}\right)=H^{1}\left(C, L \otimes K_{C}^{\vee}\right),
$$

which is well-defined up to a coefficient.

Proposition 27. The two elements e and $f$ are proportional.

Proof. Equivalently, we show that the kernels of the two linear forms $e, f \in H^{0}(C, L)^{\vee}$ are equal. Viewing $\gamma$ as an element of $\operatorname{Hom}\left(I_{C}(2)^{\vee}, H^{0}(C, L)\right)$, we have $\operatorname{Ker}(f)=\operatorname{Im}(\gamma)$. On the other hand, the kernel of $e$ identifies with

$$
\operatorname{Im}\left\{j: H^{0}\left(C, F \otimes L^{\otimes 3} \otimes K_{C}^{\vee}\right) \rightarrow H^{0}(C, L)\right\},
$$

where the map $j$ is obtained by twisting the exact sequence $0 \rightarrow \mathcal{O}_{C} \rightarrow F(L) \rightarrow K_{C} \otimes L^{\vee} \rightarrow 0$ by $K_{C}$. We have $F \otimes L^{\otimes 3} \otimes K_{C}^{\vee} \cong F^{\vee}$ since $\operatorname{det} F \cong K_{C} \otimes L^{\otimes(-3)}$, hence there is a natural morphism

$$
i^{*}: I_{C}(2)^{\vee} \otimes \mathcal{O}_{C} \rightarrow F^{\vee} \cong F\left(L^{\otimes 3} \otimes K_{C}^{\vee}\right)
$$

dual to the inclusion $F \hookrightarrow I_{C}(2) \otimes \mathcal{O}_{C}$ of (6.30). The proposition follows from the following claim:

Claim. The morphism $\alpha: I_{C}(2)^{\vee} \rightarrow H^{0}(C, L)$ is equal to $j \circ i^{*}$.

Forgetting about the last identification $F^{\vee} \cong F \otimes L^{\otimes 3} \otimes K_{C}^{\vee}$ ), the claim amounts to the following general fact: For an evaluation exact sequence on a variety $X$

$$
0 \longrightarrow G \longrightarrow W \otimes \mathcal{O}_{X} \longrightarrow M \longrightarrow 0
$$

and for a section $s \in H^{0}(X, G(L))=H^{0}\left(X, \mathcal{H o m}\left(G^{\vee}, L\right)\right)$ giving an element

$$
s^{\prime} \in \operatorname{Ker}\left\{W \otimes H^{0}(X, L) \rightarrow H^{0}(X, M \otimes L)\right\} \subseteq \operatorname{Hom}\left(W^{\vee}, H^{0}(X, L)\right),
$$

the induced map $s: H^{0}\left(X, G^{\vee}\right) \rightarrow H^{0}(X, L)$ composed with the map $W^{\vee} \rightarrow H^{0}\left(X, G^{\vee}\right)$ equals the map $s^{\prime}: W^{\vee} \rightarrow H^{0}(X, L)$. 


\section{B. Further properties}

Using the exact sequence (6.30) in the general case of a genus 8 paracanonical curve $[C, L] \in P_{8}^{14}$, we obtain:

Lemma 28. A section $s \in H^{0}(C, F(L)) \subseteq I_{C, L}(2) \otimes H^{0}(C, L)=\operatorname{Hom}\left(I_{C, L}(2)^{\vee}, H^{0}(C, L)\right)$ of rank 6 , determines an element $e \in\left|2 L-K_{C}\right|$.

Proof. The multiplication by $s \in H^{0}(F(L)) \subseteq I_{C, L}(2) \otimes H^{0}(C, L)=H^{0}\left(I_{C, L}(2)^{\vee} \otimes L\right)$ determines the natural maps $F^{\vee} \rightarrow L$ and $g_{s}: I_{C}(2)^{\vee} \otimes \mathcal{O}_{C} \rightarrow L$ sitting in the following diagram:

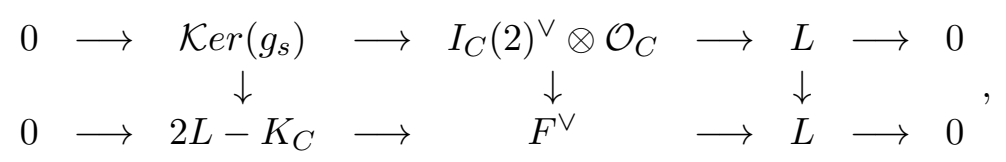

where $I_{C}(2)^{\vee} \otimes \mathcal{O}_{C} \rightarrow F^{\vee}$ is the dual of the natural inclusion of (6.30). Passing to global sections we get the inclusion $H^{0}\left(\mathcal{K} e r\left(g_{s}\right)\right)=\operatorname{Ker}\left\{I_{C, L}(2)^{\vee} \rightarrow H^{0}(C, L)\right\} \hookrightarrow H^{0}\left(2 L-K_{C}\right)$, which by hypothesis in 1-dimensional hence it defines an element $e \in\left|2 L-K_{C}\right|$.

Via the exact sequence (6.30) we can also show directly the following result that has been used in Section 3:

Lemma 29. If there is a spin curve $D=C \cup E \hookrightarrow \boldsymbol{P}^{6}$ of genus 22 and degree 21 containing the genus 8 paracanonical curve $[C, L]$ as in Lemma 3 , then $H^{0} C,(F(L)) \neq 0$. If there is no cubic polynomial on $\boldsymbol{P}^{6}$ vanishing with multiplicity 2 along $C$, then $K_{1,2}(C, L) \neq 0$.

Proof. Let $e=C \cap E$ and recall $c_{1}(F)=-3 L+K_{C}$ and $\mathcal{O}_{C}(e)=2 L-K_{C}$. Note that $I_{D}(2) \subseteq I_{C}(2)$ is 6 -dimensional. Tensor then the first vertical exact sequence of the following diagram by $L$ and pass to global sections.

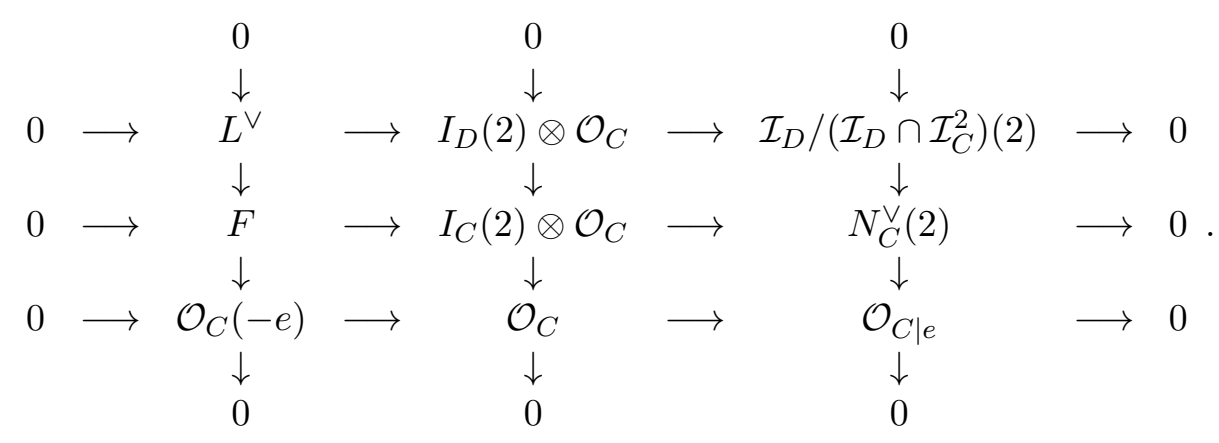

\section{C. Nontrivial syzygies of paracanonical curves via vector bundles}

We return to the proof of Theorem 20 given in Section 5. Consider now a general paracanonical curve $\left[C, K_{C} \otimes \eta\right] \in P_{8}^{14}$. For a rank 2 vector bundle on $C$ of degree 14 , with noncanonical determinant, the equation $h^{0}(C, E) \geq 4$ imposes 16 conditions. Similarly, if $\epsilon \in \operatorname{Pic}^{0}(C)$, the equation $h^{0}(C, E \otimes \epsilon) \geq 4$ imposes 16 conditions on the parameter space of $E$. Given $C$, there are $29=4 g-3$ parameters for $E$, and $8=g$ parameters for $\epsilon$. It follows that we have at least a 5 -dimensional family of pairs $(E, \epsilon)$, such that

$$
h^{0}(C, E) \geq 4 \text { and } h^{0}(C, E \otimes \epsilon) \geq 4 .
$$

Furthermore, the construction of Section 5 (together with Proposition 5) shows that for a general triple $(C, E, \epsilon)$ as above, one has $K_{2,1}(C, L) \neq 0$, where $L:=\operatorname{det} E \otimes \epsilon$. Assuming the map $(E, \epsilon) \mapsto L$ 
is generically finite on its image, we constructed in this way a five dimensional family of paracanonical line bundles $L \in \operatorname{Pic}^{14}(C)$ with a nontrivial syzygy: $K_{1,2}(C, L) \neq 0$. This family has the following property:

Lemma 30. If $L=\operatorname{det} E \otimes \epsilon$, where E satisfies (6.33), the line bundle $K_{C}^{\otimes 2} \otimes L^{\vee}$ satisfies the same property. The family above, which has dimension at least five, is thus invariant under the involution $L \mapsto K_{C}^{\otimes 2} \otimes L^{\vee}$ on $P_{8}^{14}$, whose fixed locus is the Prym moduli space $\mathcal{R}_{8}$.

Proof. This follows from Serre duality, replacing $E$ with $E^{\vee} \otimes K_{C}$ and $E \otimes \epsilon$ by $E^{\vee} \otimes \epsilon^{\vee} \otimes K_{C}$ plus the fact that $\operatorname{det}\left(E^{\vee} \otimes K_{C}\right) \otimes \epsilon^{\vee} \cong K_{C}^{\otimes 2} \otimes \operatorname{det} E^{\vee} \otimes \epsilon^{\vee}$.

One can ask in general the following question:

Question 31. Is the divisor $\mathfrak{K} \mathfrak{o s z}$ on $P_{8}^{14}$ invariant under the involution $L \mapsto K_{C}^{\otimes 2} \otimes L^{\vee}$ ?

\section{References}

[1] G. Bruns, Twists of Mukai bundles and the geometry of the level 3 modular variety over $\overline{\mathcal{M}}_{8}$, Trans. Amer. Math. Soc, to appear. arXiv:1606.07038

[2] A. Chiodo and G. Farkas, Singularities of the moduli space of level curves, J. Eur. Math. Soc. 19 (2017), no. 3, 603-658. MR-3612865

[3] A. Chiodo, D. Eisenbud, G. Farkas and F.-O Schreyer, Syzygies of torsion bundles and the geometry of the level $\ell$ modular variety over $\mathcal{M}_{g}$, Invent. Math. 194 (2013), no. 1, 73-118. MR-3103256

[4] E. Colombo and P. Frediani, On the Koszul cohomology of canonical and Prym-canonical binary curves, Bull. Lond. Math. Soc. 45 (2013), no. 5, 1031-1040. MR-3104994

[5] M. Cornalba, Moduli of curves and theta-characteristics. In: Lectures on Riemann surfaces (Trieste, 1987), pp. 560-589, World Sci. Publ., Teaneck, NJ, 1989. MR-1082361

[6] D. Eisenbud, H. Lange, G. Martens and F.-O. Schreyer, The Clifford dimension of a projective curve, Compositio Math. 72(1989), no. 2, 173-204. MR-1030141

[7] B. Eröcal, O. Motsak, F.-O. Schreyer and A. Steenpass, Refined algorithms to compute syzygies, J. Symbolic Comput. 74 (2016), 308-327. MR-3424044

[8] G. Farkas and M. Kemeny, The Generic Green-Lazarsfeld secant conjecture, Invent. Math. 203 (2016), no. 1, 265-301. MR-3437872

[9] G. Farkas and M. Kemeny, The Prym-Green Conjecture for torsion bundles of high order, Duke Math. J. 166 (2017), no. 6, 1103-1124. MR-3635900

[10] G. Farkas and K. Ludwig, The Kodaira dimension of the moduli space of Prym varieties, J. Eur. Math. Soc. 12 (2010), no. 3, 755-795. MR-2639318

[11] G. Farkas and A. Verra, Prym varieties and moduli of polarized Nikulin surfaces, Adv. Math. 290 (2016), 314-328. MR-3451926

[12] M. Green and R. Lazarsfeld, On the projective normality of complete linear series on an algebraic curve, Invent. Math. 83 (1986), no. 1, 73-90. MR-0813583 
[13] J. Harris, Theta-characteristics on algebraic curves, Trans. Amer. Math. Soc. 271 (1982), no. 2, 611-638. MR-0654853

[14] S. Mukai, Vector bundles and Brill-Noether theory. In: Current topics in complex algebraic geometry (Berkeley, CA, 1992/93), pp. 145-158, Math. Sci. Res. Inst. Publ., 28, Cambridge Univ. Press, Cambridge, 1995. MR-1397062

[15] D. Mumford, Theta characteristics of an algebraic curve, Ann. Sci. École Norm. Sup. (4) 4 (1971), 181-192. MR-0292836

[16] F.-O. Schreyer, A standard basis approach to syzygies of canonical curves, J. Reine Angew. Math. 421 (1991), 83-123. MR-1129577

[17] C. Voisin, Sur l'application de Wahl des courbes satisfaisant la condition de Brill-Noether-Petri, Acta Math. 168 (1992), no. 3-4, 249-272. MR-1161267

[18] C. Voisin, Green's generic syzygy conjecture for curves of even genus lying on a K3 surface, J. Eur. Math. Soc. 4 (2002), no. 4, 363-404. MR-1941089

[19] C. Voisin, Green's canonical syzygy conjecture for generic curves of odd genus, Compos. Math. 141 (2005), no. 5, 1163-1190. MR-2157134 